\title{
EXTREMES OF STATIONARY GAUSSIAN STORAGE MODELS
}

\author{
KRZYSZTOF DȨBICKI AND PENG LIU
}

\begin{abstract}
For the stationary storage process $\{Q(t), t \geq 0\}$, with $Q(t)=\sup _{s \geq t}\left(X(s)-X(t)-c(s-t)^{\beta}\right)$, where $\{X(t), t \geq 0\}$ is a centered Gaussian process with stationary increments, $c>0$ and $\beta>0$ is chosen such that $Q(t)$ is finite a.s., we derive exact asymptotics of $\mathbb{P}\left(\sup _{t \in\left[0, T_{u}\right]} Q(t)>u\right)$ and $\mathbb{P}\left(\inf _{t \in\left[0, T_{u}\right]} Q(t)>u\right)$, as $u \rightarrow \infty$. As a by-product we find conditions under which strong Piterbarg property holds.
\end{abstract}

Key Words: Storage process; Gaussian process; Pickands constant; strong Piterbarg property.

AMS Classification: Primary 60G15; secondary 60G70

\section{INTRODUCTION}

Let $\{X(t), t \geq 0\}$ be a centered Gaussian process with stationary increments, a.s. continuous sample paths and variance function $\sigma^{2}(t)$. Given $c>0$ and $\beta>0$, consider the stationary storage process $\{Q(t), t \geq 0\}$, with

$$
Q(t)=\sup _{s \geq t}\left(X(s)-X(t)-c(s-t)^{\beta}\right), \quad t \geq 0
$$

where $c>0$ and $\beta>0$ is chosen appropriately to guarantee a.s. finiteness of $Q(t)$.

The stimulus to analyze distributional properties of $\{Q(t), t \geq 0\}$ stems, for instance, from its straightforward relation with the theory of reflected Gaussian processes, its applications in widely investigated Gaussian fluid queueing models and, by duality, its importance in risk theory. In particular, for $\beta=1$, by Reich representation [15], $Q(t)$ describes the stationary amount of substance in reservoir, where the inflow to the reservoir in time interval $[s, t]$ equals to $X(t)-X(s)$ and the rate of outflow is $c$.

Motivated by the above applications, $Q(0)$ has been studied in the literature under different levels of generality, e.g., [12], [8], [5], [9], [6], [7], [11]. Particularly vast interest has been paid to the analysis of storage models, where $X(t)=B_{H}(t)$ is a fractional Brownian motion $(\mathrm{fBm})$ with Hurst index $H \in(0,1)$ and $\beta=1$, leading to derivation of exact asymptotics of $\mathbb{P}(Q(0)>u)$ as $u \rightarrow \infty$ in [8] and a surprising asymptotic equivalence

$$
\mathbb{P}\left(\sup _{t \in\left[0, T_{u}\right]} Q(t)>u\right) \sim \mathbb{P}(Q(0)>u) \sim \mathbb{P}\left(\inf _{t \in\left[0, T_{u}\right]} Q(t)>u\right)
$$

as $u \rightarrow \infty$, providing that $H>1 / 2$ and $T_{u}=o\left(u^{\frac{2 H-1}{H}}\right)$; see [14], [4]. Property (2) is nowadays referred to as the strong Piterbarg property. In [2] it was observed that (2) holds also for storage processes with self-similar and infinitely divisible input without Gaussian component.

Date: May 15, 2016. 
In this contribution we focus on asymptotic properties of

$$
\psi_{T_{u}}^{\sup }(u):=\mathbb{P}\left(\sup _{t \in\left[0, T_{u}\right]} Q(t)>u\right)
$$

and

$$
\psi_{T_{u}}^{\inf }(u):=\mathbb{P}\left(\inf _{t \in\left[0, T_{u}\right]} Q(t)>u\right),
$$

as $u \rightarrow \infty$, for wide classes of Gaussian processes $X$ and ranges of $T_{u}$. As a result, we extend findings of [6], where the asymptotics of $\mathbb{P}(Q(0)>u)$ was considered. Moreover we generalize [14] and [4] where the exact asymptotics of $\psi_{T_{u}}^{\mathrm{sup}}(u)$ and $\psi_{T_{u}}^{\inf }(u)$ were studied for fractional Brownian motion model with $\beta=1$. As a by-product we find conditions under which the strong Piterbarg property phenomena (2) holds for general Gaussian $X$ and $\beta$.

Organization of the paper. Some necessary notation are introduced in Section 2, whereas the main asymptotic results are presented in Section 3. In Section 4 we apply derived results to the analysis of $\psi_{T_{u}}^{\sup }(u)$ and $\psi_{T_{u}}^{\inf }(u)$ for $X$ being a sum of independent fractional Brownian motions. The proofs of main results are given in Section 5 . The Appendix contains proofs of some lemmas that are of technical nature.

\section{Notation}

Throughout this paper we assume that $\{X(t), t \geq 0\}$ is a centered Gaussian process with stationary increments, a.s. continuous sample paths, $X(0)=0$ and variance function $\sigma^{2}(t)$ satisfying

AI: $\sigma^{2}(t)>0, t>0$ is regularly varying at infinity with index $2 \alpha_{\infty} \in(0,2)$ and twice continuously differentiable on $(0, \infty)$. Further, its first derivative $\dot{\sigma}^{2}$ and second derivative $\ddot{\sigma^{2}}$ are both ultimately monotone.

AII: $\sigma^{2}(t)$ is regularly varying at 0 with index $2 \alpha_{0} \in(0,2]$.

We recall that a measurable function $f: \mathbb{R}_{+} \rightarrow \mathbb{R}_{+}$is said to be regularly varying at $\infty$ with index $\alpha \in \mathbb{R}$ if $\lim _{x \rightarrow \infty} \frac{f(\lambda x)}{f(x)}=\lambda^{\alpha}$ for any $\lambda>0$. Analogously, we say that $f$ is regularly varying at 0 with index $\alpha \in \mathbb{R}$ if for any $\lambda>0, \lim _{x \rightarrow 0} \frac{f(\lambda x)}{f(x)}=\lambda^{\alpha}$. We refer to [3] for properties of regularly varying functions. Function $f$ is ultimately monotone if there exists a constant $M>0$ such that $f(t)$ is monotone over $(M, \infty)$. Assumptions AI-AII allow us to cover models that play important role in Gaussian storage models, including both aggregations of fractional Brownian motions and integrated stationary Gaussian processes; see, e.g., [12, 8, 6, 5]. AI-AII go in line with [6], where the exact asymptotics of $\mathbb{P}(Q(0)>u)$, as $u \rightarrow \infty$, was derived.

Recall that fractional Brownian motion $B_{H}=\left\{B_{H}(t), t \geq 0\right\}$ with Hurst index $H \in(0,1]$ is a centered Gaussian process with continuous sample path and covariance function $\operatorname{Cov}\left(B_{H}(t), B_{H}(s)\right)=\frac{1}{2}\left(|t|^{2 H}+|s|^{2 H}-|t-s|^{2 H}\right)$.

For $X(t)$ satisfying AI-AII, $c>0$ and $\beta>\alpha_{\infty}$ we define the storage process $\{Q(t), t \geq 0\}$, where

$$
Q(t)=\sup _{s \geq t}\left(X(s)-X(t)-c(s-t)^{\beta}\right), \quad t \geq 0 .
$$

Note that assumption $\beta>\alpha_{\infty}$ ensures that $Q(t)$ is finite a.s. for any $t \geq 0$. 
In order to formulate the main results of this contribution, following [4], let

$$
\Phi: C(M) \rightarrow \mathbb{R}
$$

be a continuous functional on the Banach space $C(M)$ of all continuous functions on compact set $M \subset \mathbb{R}^{d}, d \geq 1$ with the norm $\|f\|=\sup _{\boldsymbol{t} \in M}|f(\boldsymbol{t})|$, satisfying

F1: $\Phi(f) \leq \sup _{\boldsymbol{t} \in M} f(\boldsymbol{t})$.

F2: $\Phi(a f+b)=a \Phi(f)+b$, for any $a>0, b \in \mathbb{R}$.

Then, for a centered continuous Gaussian field $V=\left\{V(\boldsymbol{t}): \boldsymbol{t} \in \mathbb{R}^{d}\right\}$ such that $V(\mathbf{0})=0$,

$$
\operatorname{Cov}(V(\boldsymbol{t}), V(\boldsymbol{s}))=\frac{\sigma_{V}^{2}(\boldsymbol{t})+\sigma_{V}^{2}(\boldsymbol{s})-\sigma_{V}^{2}(\boldsymbol{t}-\boldsymbol{s})}{2},
$$

we introduce the generalized Pickands' constant

$$
\mathcal{H}_{V}^{\Phi}(M)=\mathbb{E}\left(e^{\Phi\left(\sqrt{2} V-\sigma_{V}^{2}\right)}\right) .
$$

We refer to [4] for the finiteness of $\mathcal{H}_{V}^{\Phi}(M)$. In particular, for $M=\prod_{i=1}^{d}\left[0, S_{i}\right]$ with $S_{i}>0,1 \leq i \leq d$, and $\Phi(f)=\sup _{\boldsymbol{t} \in \prod_{i=1}^{d}\left[0, S_{i}\right]} f(\boldsymbol{t})$, we use notation $\mathcal{H}_{V}\left(\prod_{i=1}^{d}\left[0, S_{i}\right]\right)$. Further, for $d=1$, let

$$
\mathcal{H}_{V}=\lim _{S \rightarrow \infty} \frac{\mathcal{H}_{V}[0, S]}{S},
$$

providing that the above limit exists, where $\mathcal{H}_{V}[0, S]:=\mathcal{H}_{V}([0, S])$. We refer to [13], [5], [6] and [4] for the analysis of properties of Pickands'-type constants.

We write $f_{u}(t) \Rightarrow f(t)$ for $t \in D$ meaning that the convergence is uniform with respect to $t$ in the domain $D$ as $u \rightarrow \infty$. By $\mathbb{Q}$ and $\mathbb{Q}_{i}, i=0,1,2, \ldots$, we denote some positive constants which may change from line to line. By $\overleftarrow{\sigma}(\cdot)$ we denote the generalized inverse function to $\sigma(\cdot), \Psi(\cdot)$ denotes the tail distribution of the standard Normal random variable. We write $f(u) \sim g(u)$ if $\lim _{u \rightarrow \infty} \frac{f(u)}{g(u)}=1$.

\section{Main Results}

In this section, we present the exact asymptotics of $\psi_{T_{u}}^{\mathrm{sup}}(u)$ and $\psi_{T_{u}}^{\mathrm{inf}}(u)$. In further analysis we tacitly assume that the variance function $\sigma^{2}$ of $X$ satisfies both AI and AII.

Let

$$
\varphi:=\lim _{u \rightarrow \infty} \frac{\sigma^{2}\left(u^{1 / \beta}\right)}{u},
$$

assuming that the limit exists. As it is shown below, according to the value of $\varphi$, the asymptotics of $\psi_{T_{u}}^{\sup }(u)$ takes different form. Additionally, we introduce $\tau^{*}=\left(\frac{\alpha_{\infty}}{c\left(\beta-\alpha_{\infty}\right)}\right)^{1 / \beta}$ and set

$$
\Delta(u):=\left\{\begin{array}{cc}
\overleftarrow{\sigma}\left(\frac{\sqrt{2} \sigma^{2}\left(u^{1 / \beta} \tau^{*}\right)}{u\left(1+c \tau^{* \beta}\right)}\right), & \text { if } \varphi=\infty \text { or } 0 \\
1, & \text { if } \varphi \in(0, \infty)
\end{array}\right.
$$

Let

$$
A=\left(\frac{\alpha_{\infty}}{c\left(\beta-\alpha_{\infty}\right)}\right)^{-\alpha_{\infty} / \beta} \frac{\beta}{\beta-\alpha_{\infty}}, \quad B=\left(\frac{\alpha_{\infty}}{c\left(\beta-\alpha_{\infty}\right)}\right)^{-\left(\alpha_{\infty}+2\right) / \beta} \alpha_{\infty} \beta, \quad m^{*}(u)=\frac{u\left(1+c\left(\tau^{*}\right)^{\beta}\right)}{\sigma\left(u^{1 / \beta}\right)\left(\tau^{*}\right)^{\alpha_{\infty}}} .
$$


Theorem 3.1. Suppose that $\varphi=0$.

i) If $\frac{T_{u}}{\Delta(u)} \rightarrow \rho \in[0, \infty)$, then

$$
\psi_{T_{u}}^{\sup }(u) \sim \mathcal{H}_{B_{\alpha_{0}}}[0, \rho] \mathcal{H}_{B_{\alpha_{0}}} \sqrt{\frac{2 A \pi}{B}} \frac{u^{1 / \beta-1} \sigma\left(u^{1 / \beta} \tau^{*}\right)}{\left(1+c \tau^{* \beta}\right) \Delta(u)} \Psi\left(\inf _{t \geq 0} \frac{u\left(1+c t^{\beta}\right)}{\sigma\left(u^{1 / \beta} t\right)}\right) .
$$

ii) If $\frac{T_{u}}{\Delta(u)} \rightarrow \infty$ and $T_{u}=o\left(e^{\beta_{1}\left(m^{*}(u)\right)^{2}}\right)$ with $\beta_{1} \in(0,1 / 2)$, then

$$
\psi_{T_{u}}^{\sup }(u) \sim\left(\mathcal{H}_{B_{\alpha_{0}}}\right)^{2} \sqrt{\frac{2 A \pi}{B}} T_{u} \frac{u^{1 / \beta-1} \sigma\left(u^{1 / \beta} \tau^{*}\right)}{\left(1+c \tau^{* \beta}\right) \Delta^{2}(u)} \Psi\left(\inf _{t \geq 0} \frac{u\left(1+c t^{\beta}\right)}{\sigma\left(u^{1 / \beta} t\right)}\right) .
$$

Theorem 3.2. Suppose that $\varphi \in(0, \infty)$.

i) If $T_{u} \rightarrow \rho \in[0, \infty)$, then

$$
\psi_{T_{u}}^{\sup }(u) \sim \mathcal{H}_{\frac{1+c\left(\tau^{*}\right)^{\beta}}{\sqrt{2} \varphi\left(\tau^{*}\right)^{2 \alpha \infty}}}[0, \rho] \mathcal{H}{\frac{1+c\left(\tau^{*} \beta\right.}{\sqrt{2} \varphi\left(\tau^{*}\right)^{2 \alpha} \infty}} X \sqrt{\frac{2 A \pi}{B}} \frac{u^{1 / \beta-1} \sigma\left(u^{1 / \beta} \tau^{*}\right)}{\left(1+c \tau^{* \beta}\right)} \Psi\left(\inf _{t \geq 0} \frac{u\left(1+c t^{\beta}\right)}{\sigma\left(u^{1 / \beta} t\right)}\right) .
$$

ii) If $T_{u} \rightarrow \infty$ and $T_{u}=o\left(e^{\beta_{1}\left(m^{*}(u)\right)^{2}}\right)$ with $\beta_{1} \in(0,1 / 2)$, then

$$
\psi_{T_{u}}^{\sup }(u) \sim\left(\mathcal{H}_{\frac{1+c\left(\tau^{*} \beta\right.}{\sqrt{2} \varphi\left(\tau^{*}\right)^{2 \alpha} \infty}} X\right)^{2} \sqrt{\frac{2 A \pi}{B}} T_{u} \frac{u^{1 / \beta-1} \sigma\left(u^{1 / \beta} \tau^{*}\right)}{\left(1+c \tau^{* \beta}\right)} \Psi\left(\inf _{t \geq 0} \frac{u\left(1+c t^{\beta}\right)}{\sigma\left(u^{1 / \beta} t\right)}\right) .
$$

Theorem 3.3. Suppose that $\varphi=\infty$.

i) If $\frac{T_{u}}{\Delta(u)} \rightarrow \rho \in[0, \infty)$, then

$$
\psi_{T_{u}}^{\sup }(u) \sim \mathcal{H}_{B_{\alpha_{\infty}}}[0, \rho] \mathcal{H}_{B_{\alpha_{\infty}}} \sqrt{\frac{2 A \pi}{B}} \frac{u^{1 / \beta-1} \sigma\left(u^{1 / \beta} \tau^{*}\right)}{\left(1+c \tau^{* \beta}\right) \Delta(u)} \Psi\left(\inf _{t \geq 0} \frac{u\left(1+c t^{\beta}\right)}{\sigma\left(u^{1 / \beta} t\right)}\right) .
$$

ii) If $\frac{T_{u}}{\Delta(u)} \rightarrow \infty$ and $T_{u}=o\left(e^{\beta_{1}\left(m^{*}(u)\right)^{2}}\right)$ with $\beta_{1} \in(0,1 / 2)$, then

$$
\psi_{T_{u}}^{\sup }(u) \sim\left(\mathcal{H}_{B_{\alpha \infty}}\right)^{2} \sqrt{\frac{2 A \pi}{B}} T_{u} \frac{u^{1 / \beta-1} \sigma\left(u^{1 / \beta} \tau^{*}\right)}{\left(1+c \tau^{* \beta}\right) \Delta^{2}(u)} \Psi\left(\inf _{t \geq 0} \frac{u\left(1+c t^{\beta}\right)}{\sigma\left(u^{1 / \beta} t\right)}\right) .
$$

The above trichotomy with respect to the value of $\varphi$ goes in line with findings of Dieker [6], where the asymptotics of $\mathbb{P}(Q(0)>u)$, as $u \rightarrow \infty$, was derived.

The following theorem deals with the asymptotic behavior of the tail distribution of $\psi_{T_{u}}^{\inf }(u)$. We recall that $\mathcal{H}_{V}^{\text {inf }}[0, \rho]$ is defined according to (7).

Theorem 3.4. i) If $\varphi=0$ and $\frac{T_{u}}{\Delta(u)} \rightarrow \rho \in[0, \infty)$, then

$$
\psi_{T_{u}}^{\inf }(u) \sim \mathcal{H}_{B_{\alpha_{0}}}^{\inf }[0, \rho] \mathcal{H}_{B_{\alpha_{0}}} \sqrt{\frac{2 A \pi}{B}} \frac{u^{1 / \beta-1} \sigma\left(u^{1 / \beta} \tau^{*}\right)}{\left(1+c \tau^{* \beta}\right) \Delta(u)} \Psi\left(\inf _{t \geq 0} \frac{u\left(1+c t^{\beta}\right)}{\sigma\left(u^{1 / \beta} t\right)}\right) ;
$$

ii) If $\varphi \in(0, \infty)$ and $T_{u} \rightarrow \rho \in[0, \infty)$, then

$$
\psi_{T_{u}}^{\inf }(u) \sim \mathcal{H}_{\frac{1+c\left(\tau^{*}\right)^{\beta}}{\sqrt{2} \varphi\left(\tau^{*}\right)^{2 \alpha} \infty}}^{\inf }[0, \rho] \mathcal{H}{\frac{1+c\left(\tau^{*} \beta\right.}{\sqrt{2} \varphi\left(\tau^{*}\right)^{2 \alpha} \infty}} X \sqrt{\frac{2 A \pi}{B}} \frac{u^{1 / \beta-1} \sigma\left(u^{1 / \beta} \tau^{*}\right)}{\left(1+c \tau^{* \beta}\right)} \Psi\left(\inf _{t \geq 0} \frac{u\left(1+c t^{\beta}\right)}{\sigma\left(u^{1 / \beta} t\right)}\right) ;
$$

iii) If $\varphi=\infty$ and $\frac{T_{u}}{\Delta(u)} \rightarrow \rho \in[0, \infty)$, then

$$
\psi_{T_{u}}^{\inf }(u) \sim \mathcal{H}_{B_{\alpha_{\infty}}}^{\inf }[0, \rho] \mathcal{H}_{B_{\alpha_{\infty}}} \sqrt{\frac{2 A \pi}{B}} \frac{u^{1 / \beta-1} \sigma\left(u^{1 / \beta} \tau^{*}\right)}{\left(1+c \tau^{* \beta}\right) \Delta(u)} \Psi\left(\inf _{t \geq 0} \frac{u\left(1+c t^{\beta}\right)}{\sigma\left(u^{1 / \beta} t\right)}\right) .
$$


Combination of the above findings straightforwardly leads to the following corollary that deals with the strong Piterbarg property for $Q$, extending results derived in [4].

Corollary 3.5. Suppose that $\frac{T_{u}}{\Delta(u)} \rightarrow 0$. Then

$$
\psi_{T_{u}}^{\inf }(u) \sim \psi_{T_{u}}^{\sup }(u) \sim \psi_{0}(u)
$$

Remark 3.6. The relation $\frac{T_{u}}{\Delta(u)} \rightarrow 0$ in Corollary 3.5 is optimal. Indeed, if $\frac{T_{u}}{\Delta(u)} \rightarrow \rho>0$, then comparing Theorems 3.1, 3.2, 3.3 and 3.4, none of the asymptotic relation in Corollary 3.5 holds.

\section{Application to heterogenous fluid Queues}

Consider the stationary storage model

$$
Q(t)=\sup _{s \geq t}\left(\sum_{i=1}^{n}\left(B_{H_{i}}(s)-B_{H_{i}}(t)\right)-c(s-t)^{\beta}\right), \quad t \geq 0
$$

where $B_{H_{i}}(t), 1 \leq i \leq n$ are mutually independent fractional Brownian motions with indexes $1>H_{1}>H_{2} \geq$ $\cdots \geq H_{n-1}>H_{n}>0$ respectively and $\beta>H_{1}$. It is straightforward to check that $\sigma_{\Sigma}^{2}(t):=\operatorname{Var}\left(\sum_{i=1}^{n} B_{H_{i}}(t)\right)=$ $\sum_{i=1}^{n} t^{2 H_{i}}$ satisfies AI-AII with $\alpha_{0}=H_{n}$ and $\alpha_{\infty}=H_{1}$, which in the light of Theorems $3.1,3.2$ and 3.3 , leads to.

Corollary 4.1. Suppose that $2 H_{1}<\beta$.

i)If $T_{u} u^{\frac{\beta-2 H_{1}}{\beta H_{n}}} \sim \rho\left(\frac{\sqrt{2}\left(\tau^{*}\right)^{2 H_{1}}}{1+c\left(\tau^{*}\right)^{\beta}}\right)^{\frac{1}{H_{n}}}$ as $u \rightarrow \infty$, with $\rho \in[0, \infty)$, then

$$
\psi_{T_{u}}^{\sup }(u) \sim \mathcal{H}_{B_{H_{n}}}[0, \rho] \mathcal{H}_{B_{H_{n}}} 2^{\frac{H_{n}-1}{2 H_{n}}} \sqrt{\frac{A \pi}{B}} \frac{\left(1+c\left(\tau^{*}\right)^{\beta}\right)^{\frac{1-H_{n}}{H_{n}}}}{\left(\tau^{*}\right)^{\frac{H_{1}\left(2-H_{n}\right)}{H_{n}}}} u^{\frac{H_{n}+\beta-2 H_{1}+H_{1} H_{n}-\beta H_{n}}{\beta H_{n}}} \Psi\left(\inf _{t \geq 0} \frac{u\left(1+c t^{\beta}\right)}{\sigma_{\Sigma}\left(u^{1 / \beta} t\right)}\right)
$$

ii) If $T_{u} u^{\frac{\beta-2 H_{1}}{\beta H_{n}}} \rightarrow \infty$ and $T_{u}=o\left(e^{\beta_{1}\left(m^{*}(u)\right)^{2}}\right)$ with $\beta_{1} \in(0,1 / 2)$, then

$$
\psi_{T_{u}}^{\sup }(u) \sim\left(\mathcal{H}_{B_{H_{n}}}\right)^{2} 2^{\frac{H_{n}-2}{2 H_{n}}} \sqrt{\frac{A \pi}{B}} \frac{\left(1+c\left(\tau^{*}\right)^{\beta}\right)^{\frac{2-H_{n}}{H_{n}}}}{\left(\tau^{*}\right)^{\frac{H_{1}\left(4-H_{n}\right)}{H_{n}}}} T_{u} u^{\frac{H_{n}+2 \beta-4 H_{1}+H_{1} H_{n}-\beta H_{n}}{\beta H_{n}}} \Psi\left(\inf _{t \geq 0} \frac{u\left(1+c t^{\beta}\right)}{\sigma_{\Sigma}\left(u^{1 / \beta} t\right)}\right)
$$

Corollary 4.2. Suppose that $2 H_{1}=\beta$.

i) If $T_{u} \rightarrow \rho \in[0, \infty)$, then

$$
\psi_{T_{u}}^{\sup }(u) \sim \mathcal{H}_{\frac{1+c\left(\tau^{*}\right)^{\beta}}{\sqrt{2}\left(\tau^{*}\right)^{2 H}} \sum_{i=1}^{n} B_{H_{i}}}[0, \rho] \mathcal{H}_{\frac{1+c\left(\tau^{*}\right)^{\beta}}{\sqrt{2}\left(\tau^{*}\right)^{2 H}} \sum_{i=1}^{n} B_{H_{i}}} \sqrt{\frac{2 A \pi}{B}} \frac{\left(\tau^{*}\right)^{H_{1}}}{\left(1+c \tau^{* \beta}\right)} u^{\frac{1-H_{1}}{2 H_{1}}} \Psi\left(\inf _{t \geq 0} \frac{u\left(1+c t^{\beta}\right)}{\sigma_{\Sigma}\left(u^{1 / \beta} t\right)}\right)
$$

ii) If $T_{u} \rightarrow \infty$ and $T_{u}=o\left(e^{\beta_{1}\left(m^{*}(u)\right)^{2}}\right)$ with $\beta_{1} \in(0,1 / 2)$, then

$$
\psi_{T_{u}}^{\sup }(u) \sim\left(\mathcal{H}_{\frac{1+c\left(\tau^{*}\right)^{\beta}}{\sqrt{2}\left(\tau^{*}\right)^{2 H}} \sum_{i=1}^{n} B_{H_{i}}}\right)^{2} \sqrt{\frac{2 A \pi}{B}} \frac{\left(\tau^{*}\right)^{H_{1}}}{\left(1+c \tau^{* \beta}\right)} T_{u} u^{\frac{1-H_{1}}{2 H_{1}}} \Psi\left(\inf _{t \geq 0} \frac{u\left(1+c t^{\beta}\right)}{\sigma_{\Sigma}\left(u^{1 / \beta} t\right)}\right) .
$$

Corollary 4.3. Suppose that $2 H_{1}>\beta>H_{1}$.

i) If $T_{u} u^{\frac{\beta-2 H_{1}}{\beta H_{1}}} \rightarrow \rho\left(\frac{\sqrt{2}\left(\tau^{*}\right)^{2 H_{1}}}{1+c\left(\tau^{*}\right)^{\beta}}\right)^{\frac{1}{H_{1}}}$, with $\rho \in[0, \infty)$, then

$$
\psi_{T_{u}}^{\sup }(u) \sim \mathcal{H}_{B_{H_{1}}}[0, \rho] \mathcal{H}_{B_{H_{1}}} 2^{\frac{H_{1}-1}{2 H_{1}}} \sqrt{\frac{A \pi}{B}} \frac{\left(1+c\left(\tau^{*}\right)^{\beta}\right)^{\frac{1-H_{1}}{H_{1}}}}{\left(\tau^{*}\right)^{2-H_{1}}} u^{\frac{\left(\beta-H_{1}\right)\left(1-H_{1}\right)}{\beta H_{1}}} \Psi\left(\inf _{t \geq 0} \frac{u\left(1+c t^{\beta}\right)}{\sigma_{\Sigma}\left(u^{1 / \beta} t\right)}\right)
$$


ii) If $T_{u} u^{\frac{\beta-2 H_{1}}{\beta H_{1}}} \rightarrow \infty$ and $T_{u}=o\left(e^{\beta_{1}\left(m^{*}(u)\right)^{2}}\right)$ with $\beta_{1} \in(0,1 / 2)$, then

$$
\psi_{T_{u}}^{\sup }(u) \sim\left(\mathcal{H}_{B_{H_{1}}}\right)^{2} 2^{\frac{H_{1}-2}{2 H_{1}}} \sqrt{\frac{A \pi}{B}} \frac{\left(1+c\left(\tau^{*}\right)^{\beta}\right)^{\frac{2-H_{1}}{H_{1}}}}{\left(\tau^{*}\right)^{4-H_{1}}} T_{u} u^{\frac{2 \beta-3 H_{1}+H_{1}^{2}-\beta H_{1}}{\beta H_{1}}} \Psi\left(\inf _{t \geq 0} \frac{u\left(1+c t^{\beta}\right)}{\sigma_{\Sigma}\left(u^{1 / \beta} t\right)}\right) .
$$

Remarks 4.4. Following [4] and [14], if $n=1, H_{1}>1 / 2, \beta=1$ and $T_{u}=o\left(u^{\frac{2 H_{1}-1}{H_{1}}}\right)$, then $\psi_{T_{u}}^{\sup }(u) \sim \psi_{T_{u}}^{\inf }(u)$. $A$ straightforward application of Corollary 3.5 to the model considered in this section provides that $\psi_{T_{u}}^{\text {sup }}(u) \sim \psi_{T_{u}}^{\text {inf }}(u)$ for $n \geq 1,2 H_{1}>\beta>H_{1}$ and $T_{u}=o\left(u^{\frac{2 H_{1}-\beta}{\beta H_{1}}}\right)$.

\section{Proofs}

In this section we present detailed proofs of the main results of this contribution. Let $\tau_{u}$ be the maximizer of $\frac{\sigma\left(u^{1 / \beta} t\right)}{1+c t^{\beta}}$ over $t \geq 0$. In Lemma 5.3, we prove that $\tau_{u}$ is unique for $u$ large enough and $\lim _{u \rightarrow \infty} \tau_{u} \rightarrow \tau^{*}=\left(\frac{\alpha_{\infty}}{c\left(\beta-\alpha_{\infty}\right)}\right)^{1 / \beta}$. Following the same line of reasoning as in [14], we write

$$
\psi_{T_{u}}^{\sup }(u)=\mathbb{P}\left(\sup _{t \in\left[0, T_{u}\right]} Q(t)>u\right)=\mathbb{P}\left(\sup _{t \in\left[0, u^{-1 / \beta} T_{u}\right]} \sup _{s \geq t} Z_{u}(s, t)>m(u)\right)
$$

with $Z_{u}(s, t)=\frac{X\left(u^{1 / \beta} s\right)-X\left(u^{1 / \beta} t\right)}{1+c(s-t)^{\beta}} \frac{1+c \tau_{u}^{\beta}}{\sigma\left(u^{1 / \beta} \tau_{u}\right)}$ and $m(u)=\inf _{t \geq 0} \frac{u\left(1+c t^{\beta}\right)}{\sigma\left(u^{1 / \beta} t\right)}$.

Hereafter, for a given process $Y(t)$, we denote $\bar{Y}(t):=Y(t) / \sigma_{Y}(t)$. By $\dot{h}, \ddot{h}$ we mean the first and second derivative of twice continuously differentiable function $h$, respectively. To shorten the notation we set $\sigma_{u}^{2}(s)=\mathbb{E}\left(\frac{X\left(u^{1 / \beta} s\right)}{\sigma\left(u^{1 / \beta}\right)\left(1+c s^{\beta}\right)}\right)^{2}$ and $r_{u}\left(s, t, s_{1}, t_{1}\right):=\operatorname{Cov}\left(\overline{Z_{u}}(s, t), \overline{Z_{u}}\left(s_{1}, t_{1}\right)\right)=\mathbb{E}\left(\frac{X\left(u^{1 / \beta} s\right)-X\left(u^{1 / \beta} t\right)}{\sigma\left(u^{1 / \beta}(s-t)\right)} \frac{X\left(u^{1 / \beta} s_{1}\right)-X\left(u^{1 / \beta} t_{1}\right)}{\sigma\left(u^{1 / \beta}\left(s_{1}-t_{1}\right)\right)}\right), s>t, s_{1}>t_{1}$.

The following lemma slightly extends Lemma 2 in [6], by providing asymptotics for the tail distribution of functionals introduced in (5) fulfilling F1-F2 instead of sup functional considered in [6]. Following the setting given in [6], let $\left\{K_{u}\right\}$ be a nondecreasing family of subsets of $\mathbb{Z}^{m}$ with $m \geq 1$, and $\left\{X^{(u, \boldsymbol{k})}(\boldsymbol{t}), \boldsymbol{t} \in M\right\}, u>0, \boldsymbol{k} \in K_{u}$ be a collection of centered continuous Gaussian fields on a compact set $M \subset \mathbb{R}^{d}$ such that $0 \in M$. We assume that the variance of $X^{(u, \boldsymbol{k})}(\boldsymbol{t})$ equals 1 . Let $g_{\boldsymbol{k}}, \theta_{\boldsymbol{k}}$, with $\boldsymbol{k} \in K_{u}$ be such that (see [6])

$\mathbf{P 1} \inf _{\boldsymbol{k} \in K_{u}} g_{\boldsymbol{k}}(u) \rightarrow \infty$ as $u \rightarrow \infty$.

P2 There exists a centered Gaussian field $\left\{V(\boldsymbol{t}), \boldsymbol{t} \in \mathbb{R}^{d}\right\}$ with covariance as in (6), satisfying E1, such that $\sup _{\boldsymbol{k} \in K_{u}}\left|\theta_{\boldsymbol{k}}(u, \boldsymbol{s}, \boldsymbol{t})-\sigma_{V}^{2}(\boldsymbol{t}-\boldsymbol{s})\right| \rightarrow 0$ for any $\boldsymbol{s}, \boldsymbol{t} \in M$.

P3 For some $\eta_{1}, \cdots, \eta_{d}>0$,

$$
\limsup _{u \rightarrow \infty} \sup _{\boldsymbol{k} \in K_{u}} \sup _{\boldsymbol{s}, \boldsymbol{t} \in M, \boldsymbol{s} \neq \boldsymbol{t}} \frac{\theta_{\boldsymbol{k}}(u, \boldsymbol{s}, \boldsymbol{t})}{\sum_{i=1}^{d}\left|s_{i}-t_{i}\right|^{\eta_{i}}}<\infty
$$

$\mathbf{P} 4 \lim _{\epsilon \rightarrow 0} \limsup _{u \rightarrow \infty} \sup _{\boldsymbol{k} \in K_{u}} \sup _{|\boldsymbol{s}-\boldsymbol{t}|<\epsilon, \boldsymbol{s}, \boldsymbol{t} \in M} g_{\boldsymbol{k}}^{2}(u) \mathbb{E}\left(\left(X^{(u, \boldsymbol{k})}(\boldsymbol{s})-X^{(u, \boldsymbol{k})}(\boldsymbol{t})\right) X^{(u, \boldsymbol{k})}(\mathbf{0})\right)=0$.

Lemma 5.1. Suppose that P1-P4 hold for functions $g_{\boldsymbol{k}}, \theta_{\boldsymbol{k}}$ and Gaussian process $V$. Let $\Phi: C(M) \rightarrow \mathbb{R}$ be a continuous functional fulfiling $\mathbf{F 1}$ and $\mathbf{F 2}$. If

$$
\lim _{u \rightarrow \infty} \sup _{\boldsymbol{k} \in K_{u}} \sup _{\boldsymbol{s}, \boldsymbol{t} \in M, \boldsymbol{s} \neq \boldsymbol{t}}\left|g_{\boldsymbol{k}}^{2}(u) \frac{\operatorname{Var}\left(X^{(u, \boldsymbol{k})}(\boldsymbol{t})-X^{(u, \boldsymbol{k})}(\boldsymbol{s})\right)}{2 \theta_{\boldsymbol{k}}(u, \boldsymbol{s}, \boldsymbol{t})}-1\right|=0,
$$


then

$$
\lim _{u \rightarrow \infty} \frac{\mathbb{P}\left(\Phi\left(X^{(u, k)}\right)>g_{\boldsymbol{k}}(u)\right)}{\Psi\left(g_{\boldsymbol{k}}(u)\right)}=\mathcal{H}_{V}^{\Phi}(M)
$$

provided that $\mathbb{P}\left(\Phi\left(X^{(u, \boldsymbol{k})}\right)>g_{\boldsymbol{k}}(u)\right)>0$ for $u$ large enough, and

$$
\limsup _{u \rightarrow \infty} \sup _{\boldsymbol{k} \in K_{u}} \frac{\mathbb{P}\left(\Phi\left(X^{(u, \boldsymbol{k})}\right)>g_{\boldsymbol{k}}(u)\right)}{\Psi\left(g_{\boldsymbol{k}}(u)\right)}<\infty .
$$

The proof of Lemma 5.1 goes line-by line the same as the proof of Lemma 2 in [6]; see also proof of Lemma 1 in [4]. We present main steps of the proof in Appendix.

Lemma 5.2. Suppose that $\sigma^{2}(t)$ satisfies AI-AII. Then there exisit $\gamma \in(0,2), C_{1}>0$ and $C_{2}>0$ such that

$$
C_{1} t^{2} \leq \sigma^{2}(t) \leq C_{2} t^{\gamma}
$$

holds in a neighbourhood of zero.

Lemma 5.3. For $u$ large enough, $\sigma_{u}(\tau)$ attains its unique maximum at $\tau_{u} \in[0, \infty)$ so that $\tau_{u} \rightarrow \tau^{*}$, as $u \rightarrow \infty$. Moreover,

$$
\frac{\sigma_{u}(\tau)}{\sigma_{u}\left(\tau_{u}\right)}=1-b_{u}\left(\tau-\tau_{u}\right)^{2}(1+o(1)), \quad \tau \rightarrow \tau_{u},
$$

where $b_{u} \rightarrow b=\frac{B}{2 A}$.

Let $E(u)=\left(\tau_{u}-\delta_{u}, \tau_{u}+\delta_{u}\right)$ with $\delta_{u}=\frac{\ln (m(u))}{m(u)}$.

Lemma 5.4. The correlation function $r_{u}\left(s, t, s_{1}, t_{1}\right)$ satisfies

$$
\lim _{u \rightarrow \infty} \sup _{\left|t-t_{1}\right|<\delta_{u}, s-t, s_{1}-t_{1} \in E(u),(s, t) \neq\left(s_{1}, t_{1}\right)}\left|\frac{1-r_{u}\left(s, t, s_{1}, t_{1}\right)}{\frac{\sigma^{2}\left(u^{1 / \beta}\left|s-s_{1}\right|\right)+\sigma^{2}\left(u^{1 / \beta}\left|t-t_{1}\right|\right)}{2 \sigma^{2}\left(u^{1 / \beta} \tau^{*}\right)}}-1\right|=0 .
$$

Lemma 5.5. For $u$ large enough and any $\delta>0$, there exists a constant $0<a_{\delta}<1$ such that

$$
r_{u}\left(s, t, s_{1}, t_{1}\right)<a_{\delta}
$$

holds for all $\left|t-t_{1}\right|>\delta, s-t, s_{1}-t_{1} \in E(u)$. Further,

$$
\lim _{R \rightarrow \infty} \sup _{\left|t-t_{1}\right|>R, s-t, s_{1}-t_{1} \in E(u)} r_{u}\left(s, t, s_{1}, t_{1}\right)=0,
$$

holds uniformly with respect to $u$ for u large enough.

We provide complete proofs of Lemma 5.1, 5.2, 5.3, 5.4 and 5.5 in the Appendix.

The following lemma deals with the asymptotics of supremum of Gaussian field $Z_{u}(s, t)$ over a parameter set that is away from the neighborhood of the maximizer of the variance of $Z_{u}(s, t)$.

Recall that

$$
\Delta(u)=\left\{\begin{array}{cc}
\overleftarrow{\sigma}\left(\frac{\sqrt{2} \sigma^{2}\left(u^{1 / \beta} \tau^{*}\right)}{u\left(1+c \tau^{* \beta}\right)}\right), & \text { if } \varphi=\infty \text { or } 0 \\
1, & \text { if } \varphi \in(0, \infty)
\end{array}\right.
$$


Lemma 5.6. Suppose that AI-AII hold and $r>0$.

i) If $T_{u}=o\left(u^{r}\right)$, then

$$
\mathbb{P}\left(\sup _{t \in\left[0, T_{u} u^{-1 / \beta}\right]} \sup _{s \geq t, s-t \notin E(u)} Z_{u}(s, t)>m(u)\right)=o\left(\frac{u^{1 / \beta}}{\Delta(u) m(u)} \Psi(m(u))\right) .
$$

ii) If $u^{r} T_{u} \rightarrow \infty$, as $u \rightarrow \infty$, then

$$
\mathbb{P}\left(\sup _{t \in\left[0, T_{u} u^{-1 / \beta}\right]} \sup _{s \geq t, s-t \notin E(u)} Z_{u}(s, t)>m(u)\right)=o\left(\frac{T_{u} u^{1 / \beta}}{(\Delta(u))^{2} m(u)} \Psi(m(u))\right) .
$$

Proof. We set $\tau=s-t$ and write

$$
\mathbb{P}\left(\sup _{t \in\left[0, T_{u} u^{-1 / \beta}\right]} \sup _{s-t \notin E(u)} Z_{u}(s, t)>m(u)\right)=\mathbb{P}\left(\sup _{t \in\left[0, T_{u} u^{-1 / \beta}\right] \tau \notin E(u)} \sup _{u}(t+\tau, t)>m(u)\right) .
$$

Let $\left[0, T_{u} u^{-1 / \beta}\right] \times([0, \infty) \backslash E(u))=\mathcal{S}_{1, u} \cup \mathcal{S}_{2, u} \cup \mathcal{S}_{3, u}$, where $\mathcal{S}_{1, u}=\left[0, T_{u} u^{-1 / \beta}\right] \times[0, \epsilon], \mathcal{S}_{2, u}=\left[0, T_{u} u^{-1 / \beta}\right] \times[T, \infty)$ and $\mathcal{S}_{3, u}=\left[0, T_{u} u^{-1 / \beta}\right] \times([\epsilon, T] \backslash E(u))$, for sufficiently small $\epsilon>0$ and large $T \in \mathbb{N}$. Clearly it suffices to find the asymptotic upper estimates of the analyzed tail probability for each set $\mathcal{S}_{1, u}, \mathcal{S}_{2, u}, \mathcal{S}_{3, u}$ separately.

Ad. $\mathcal{S}_{2, u}$. Following Potter's theorem (see, e.g., [3]), for $u$ large enough,

$$
\begin{aligned}
\mathbb{E}\left(Z_{u}^{2}(t+\tau, t)\right) & =\frac{\sigma^{2}\left(u^{1 / \beta} \tau\right)\left(1+c \tau_{u}^{\beta}\right)^{2}}{\sigma^{2}\left(u^{1 / \beta} \tau_{u}\right)\left(1+c \tau^{\beta}\right)^{2}} \leq 2\left(\frac{\tau}{\tau_{u}}\right)^{2 \alpha_{\infty}+2 \eta}\left(\frac{1+c \tau_{u}^{\beta}}{1+c \tau^{\beta}}\right)^{2} \leq 2 \frac{\left(1+c \tau_{u}^{\beta}\right)^{2}}{c^{2} \tau_{u}^{2 \alpha_{\infty}+2 \eta} \tau^{2 \alpha_{\infty}+2 \eta-2 \beta}} \\
& \leq \mathbb{Q} \tau^{-2\left(\beta-\alpha_{\infty}-\eta\right)}
\end{aligned}
$$

where $\mathbb{Q}$ is a fixed constant and $0<\eta<\beta-\alpha_{\infty}$. Note that by AI and Lemma 5.2, we can choose $0<\gamma_{1}<$ $\min \left(\gamma, 2 \alpha_{\infty}\right)$ such that

$$
g_{1}(t):=\frac{\sigma^{2}(t)}{t^{\gamma_{1}}}
$$

is a regularly varying function at $\infty$ with index $2 \alpha_{\infty}-\gamma_{1}>0$ and bounded in a neighborhood of 0 . Therefore it follows from Uniform Convergence Theorem (UCT) (see, e.g., Theorem 1.5.2 in [3]) that for $t, t_{1} \in[l, l+1] \subset\left[0, T_{u} u^{-1 / \beta}+1\right]$, $\tau, \tau_{1} \in[k, k+1] \subset[T, \infty)$ and $u$ large enough,

$$
\begin{aligned}
\mathbb{E} & \left(\bar{Z}_{u}(t+\tau, t)-\bar{Z}_{u}\left(t_{1}+\tau_{1}, t_{1}\right)\right)^{2} \\
& \leq 2 \frac{\sigma^{2}\left(u^{1 / \beta}\left|t-t_{1}\right|\right)+\sigma^{2}\left(u^{1 / \beta}\left|t+\tau-t_{1}-\tau_{1}\right|\right)}{\sigma\left(u^{1 / \beta} \tau\right) \sigma\left(u^{1 / \beta} \tau_{1}\right)} \\
& \leq 4 \frac{g_{1}\left(u^{1 / \beta}\left|t-t_{1}\right|\right)}{g_{1}\left(u^{1 / \beta} k\right)}\left(\frac{\left|t-t_{1}\right|}{k}\right)^{\gamma_{1}}+4 \frac{g_{1}\left(u^{1 / \beta}\left|t+\tau-t_{1}-\tau_{1}\right|\right)}{g_{1}\left(u^{1 / \beta} k\right)}\left(\frac{\left|t+\tau-t_{1}-\tau_{1}\right|}{k}\right)^{\gamma_{1}} \\
& \leq \mathbb{Q}_{1}\left(\left|t-t_{1}\right|^{\gamma_{1}}+\left|t+\tau-t_{1}-\tau_{1}\right|^{\gamma_{1}}\right) \leq \mathbb{Q}_{2}\left(\left|t-t_{1}\right|^{\gamma_{1}}+\left|\tau-\tau_{1}\right|^{\gamma_{1}}\right),
\end{aligned}
$$

where $\mathbb{Q}_{1}, \mathbb{Q}_{2}>0$. Combining (14) and (16) with Fernique inequality (see [10]), we have, for $u$ and $T$ large enough,

$$
\begin{aligned}
& \mathbb{P}\left(\sup _{t \in\left[0, T_{u} u^{-1 / \beta}\right]} \sup _{\tau \in[T, \infty)} Z_{u}(t+\tau, t)>m(u)\right) \\
& \leq \sum_{l=0}^{\left[T_{u} u^{-1 / \beta}\right]} \sum_{k=T}^{\infty} \mathbb{P}\left(\sup _{t \in[l, l+1]} \sup _{\tau \in[k, k+1]} Z_{u}(t+\tau, t)>m(u)\right)
\end{aligned}
$$




$$
\begin{aligned}
& \leq \sum_{l=0}^{\left[T_{u} u^{-1 / \beta}\right]} \sum_{k=T}^{\infty} \mathbb{P}\left(\sup _{t \in[l, l+1]} \sup _{\tau \in[k, k+1]} \bar{Z}_{u}(t+\tau, t)>m(u) \frac{k^{\left(\beta-\alpha_{\infty}-\eta\right)}}{\sqrt{\mathbb{Q}}}\right) \\
& \leq \sum_{l=0}^{\left[T_{u} u^{-1 / \beta}\right]} \sum_{k=T}^{\infty} 16 \exp \left(-\frac{k^{2\left(\beta-\alpha_{\infty}-\eta\right)}}{\mathbb{Q}_{3}} m^{2}(u)\right) \\
& \leq \mathbb{Q}_{T}\left(\left[T_{u} u^{-1 / \beta}\right]+1\right) \exp \left(-\frac{T^{2\left(\beta-\alpha_{\infty}-\eta\right)}}{\mathbb{Q}_{3}} m^{2}(u)\right)
\end{aligned}
$$

with $\mathbb{Q}_{3}, \mathbb{Q}_{T}>0$.

Ad. $\mathcal{S}_{1, u}$. It is convenient to bound

$$
\mathbb{P}\left(\sup _{t \in\left[0, T_{u} u^{-1 / \beta}\right], \tau \in[0, \epsilon]} Z_{u}(t+\tau, t)>m(u)\right) \leq \mathbb{P}\left(\sup _{t \in\left[0, T_{u} u^{-1 / \beta}\right], \tau \in[0, \epsilon]} Z_{u}(t+\tau, t)\left(1+c \tau^{\beta}\right)>m(u)\right) .
$$

Indeed the same lines of reasoning as above leads for $t, t_{1} \in[l, l+1] \subset\left[0, T_{u} u^{-1 / \beta}+1\right], \tau, \tau_{1} \in[0, \epsilon]$ and $u$ large enough, to

$$
\begin{aligned}
\mathbb{E} & \left(Z_{u}(t+\tau, t)\left(1+c \tau^{\beta}\right)-Z_{u}\left(t_{1}+\tau_{1}, t_{1}\right)\left(1+c \tau_{1}^{\beta}\right)\right)^{2} \\
& =\frac{\left(1+c \tau_{u}^{\beta}\right)^{2}}{\sigma^{2}\left(u^{1 / \beta} \tau_{u}\right)} \mathbb{E}\left(X\left(u^{1 / \beta}(t+\tau)\right)-X\left(u^{1 / \beta} t\right)-X\left(u^{1 / \beta}\left(t_{1}+\tau_{1}\right)\right)+X\left(u^{1 / \beta} t_{1}\right)\right)^{2} \\
& \leq 2\left(1+c \tau_{u}^{\beta}\right)^{2} \frac{\sigma^{2}\left(u^{1 / \beta}\left|t-t_{1}\right|\right)+\sigma^{2}\left(u^{1 / \beta}\left|t+\tau-t_{1}-\tau_{1}\right|\right)}{\sigma^{2}\left(u^{1 / \beta} \tau_{u}\right)} \\
& =2\left(1+c \tau_{u}^{\beta}\right)^{2}\left(\frac{g_{1}\left(u^{1 / \beta}\left|t-t_{1}\right|\right)}{g_{1}\left(u^{1 / \beta} \tau_{u}\right)} \frac{\left|t-t_{1}\right|^{\gamma_{1}}}{\tau_{u}^{\gamma_{1}}}+\frac{g_{1}\left(u^{1 / \beta}\left|t+\tau-t_{1}-\tau_{1}\right|\right)}{g_{1}\left(u^{1 / \beta} \tau_{u}\right)} \frac{\left|t+\tau-t_{1}-\tau_{1}\right|^{\gamma_{1}}}{\tau_{u}^{\gamma_{1}}}\right) \\
& \leq \mathbb{Q}_{4}\left(\left|t-t_{1}\right|^{\gamma_{1}}+\left|\tau-\tau_{1}\right|^{\gamma_{1}}\right),
\end{aligned}
$$

where $\mathbb{Q}_{4}>0$. Therefore, by Fernique inequality, $\mathbb{P}\left(\sup _{t \in[l, l+1], \tau \in[0, \epsilon]} Z_{u}(t+\tau, t)\left(1+c \tau^{\beta}\right)>x\right) \leq 8 \exp \left(-\frac{x^{2}}{\mathbb{Q}_{5}}\right)$, for all $[l, l+1] \subset\left[0, T_{u} u^{-1 / \beta}+1\right]$ and $x>0$. Hence we can find a common $a>0$ such that $\mathbb{P}\left(\sup _{t \in[l, l+1], \tau \in[0, \epsilon]} Z_{u}(t+\tau, t)\left(1+c \tau^{\beta}\right)>a\right)<1 / 2$, for all $[l, l+1] \subset\left[0, T_{u} u^{-1 / \beta}+1\right]$. Moreover, we have, for $u$ large enough,

$$
\sup _{\tau \in[0, \epsilon]} \mathbb{E}\left(Z_{u}(t+\tau, t)\left(1+c \tau^{\beta}\right)\right)^{2} \leq \sup _{\tau \in[0, \epsilon]} \frac{\sigma^{2}\left(u^{1 / \beta} \tau\right)\left(1+c \tau_{u}^{\beta}\right)^{2}}{\sigma^{2}\left(u^{1 / \beta} \tau_{u}\right)} \leq \mathbb{Q}_{6}\left(\frac{\epsilon}{\tau^{*}}\right)^{2 \alpha_{\infty}} .
$$

Thus, by Borell theorem, we have that for $\epsilon$ small enough and $u$ large enough,

$$
\begin{aligned}
& \mathbb{P}\left(\sup _{t \in\left[0, T_{u} u^{-1 / \beta}\right], \tau \in[0, \epsilon]} Z_{u}(t+\tau, t)\left(1+c \tau^{\beta}\right)>m(u)\right) \\
& \leq \sum_{l=0}^{\left[T_{u} u^{-1 / \beta}\right]} \mathbb{P}\left(\sup _{t \in[l, l+1], \tau \in[0, \epsilon]} Z_{u}(t+\tau, t)\left(1+c \tau^{\beta}\right)>m(u)\right) \\
& \quad \leq 2\left(\left[T_{u} u^{-1 / \beta}\right]+1\right) \Psi\left(\frac{m(u)-a}{\sqrt{\mathbb{Q}_{6}}\left(\frac{\epsilon}{\tau^{*}}\right)^{\alpha_{\infty}}}\right),
\end{aligned}
$$

Ad. $\mathcal{S}_{3, u}$. Similarly as for $\mathcal{S}_{2, u}$, we have

$$
\mathbb{E}\left(\bar{Z}_{u}(t+\tau, t)-\bar{Z}_{u}\left(t_{1}+\tau_{1}, t_{1}\right)\right)^{2} \leq \mathbb{Q}_{7}\left(\left|t-t_{1}\right|^{\gamma_{1}}+\left|\tau-\tau_{1}\right|^{\gamma_{1}}\right),
$$


for $t, t_{1} \in[l, l+1] \subset\left[0, T_{u} u^{-1 / \beta}+1\right]$ and $\tau, \tau_{1} \in[\epsilon, T]$. Thus by Piterbarg inequality (Theorem 8.1 in [13]) and (12), for any $\epsilon>0$, we have, for $u$ sufficiently large,

$$
\begin{aligned}
& \mathbb{P}\left(\sup _{(t, \tau) \in\left[0, T_{u} u^{-1 / \beta}\right] \times([\epsilon, T] \backslash E(u))} Z_{u}(t+\tau, t)>m(u)\right) \\
& \leq \mathbb{P}\left(\sup _{(t, \tau) \in\left[0, T_{u} u^{-1 / \beta}\right] \times[\epsilon, T]} \bar{Z}_{u}(t+\tau, t)>\frac{m(u)}{1-\frac{b}{2}\left(\frac{\ln m(u)}{m(u)}\right)^{2}}\right) \\
& \leq \mathbb{Q}_{8}\left(\left[T_{u} u^{-1 / \beta}\right]+1\right) T(m(u))^{4 / \gamma_{1}} \Psi\left(\frac{m(u)}{1-\frac{b}{2}\left(\frac{\ln m(u)}{m(u)}\right)^{2}}\right) .
\end{aligned}
$$

In order to confirm i), suppose that $T_{u}=o\left(u^{r}\right)$. By the fact that $m(u)$ is regularly varying at $\infty$ with index $1-\alpha_{\infty}>0$, for $T$ sufficiently large and $\epsilon$ sufficiently small, terms (17),(19),(20) are $o\left(u^{-M} \Psi(m(u))\right)$ for any $M>0$, as $u \rightarrow \infty$, which implies that i) holds.

Analogously, under $u^{r} T_{u} \rightarrow \infty$, terms (17),(19),(20) are $o\left(u^{-M} T_{u} \Psi(m(u))\right)$ for any $M>0$, as $u \rightarrow \infty$, which establishes claim ii).

\subsection{Proof of Theorem 3.1. Since}

$$
\pi_{T_{u}}(u) \leq \psi_{T_{u}}^{\sup }(u) \leq \pi_{T_{u}}(u)+\mathbb{P}\left(\sup _{t \in\left[0, T_{u} u^{-1 / \beta}\right] s \geq t, s-t \notin E(u)} \sup _{u}(s, t)>m(u)\right),
$$

where

$$
\pi_{T_{u}}(u)=\mathbb{P}\left(\sup _{t \in\left[0, T_{u} u^{-1 / \beta}\right]} \sup _{s-t \in E(u)} Z_{u}(s, t)>m(u)\right),
$$

and the upper bound of $\mathbb{P}\left(\sup _{t \in\left[0, T_{u} u^{-1 / \beta}\right]} \sup _{s \geq t, s-t \notin E(u)} Z_{u}(s, t)>m(u)\right)$ is given in Lemma 5.6, then it suffices to focus on the asymptotics of $\pi_{T_{u}}(u)$. Since $\frac{u^{1-1 / \beta}}{\sigma\left(u^{1 / \beta}\right)} \overleftarrow{\sigma}\left(\frac{\sigma^{2}\left(u^{1 / \beta}\right)}{u}\right)$ is regularly varying at $\infty$ with index

$$
\vartheta=\left\{\begin{array}{cc}
1-1 / \beta-\alpha_{\infty} / \beta+2 \alpha_{\infty} /\left(\alpha_{0} \beta\right)-1 / \alpha_{0}, & \text { if } \varphi=0, \\
1-1 / \beta-\alpha_{\infty} / \beta, & \text { if } \varphi \in(0, \infty), \\
1+1 / \beta-\alpha_{\infty} / \beta-1 / \alpha_{\infty}, & \text { if } \varphi=\infty,
\end{array}\right.
$$

and for all $\varphi \in[0, \infty]$ we have $\vartheta<0$, then

$$
\lim _{u \rightarrow \infty} \frac{u^{1-1 / \beta}}{\sigma\left(u^{1 / \beta}\right)} \overleftarrow{\sigma}\left(\frac{\sigma^{2}\left(u^{1 / \beta}\right)}{u}\right)=0
$$

which implies that, independently of the value of $\varphi$,

$$
\lim _{u \rightarrow \infty} \frac{m(u) \Delta(u)}{u^{1 / \beta}} \rightarrow 0
$$

Let $D_{k}(u)=\left[k \frac{\Delta(u)}{u^{1 / \beta}} S,(k+1) \frac{\Delta(u)}{u^{1 / \beta}} S\right], F_{l}(u)=\left[\tau_{u}+l \frac{\Delta(u)}{u^{1 / \beta}} S, \tau_{u}+(l+1) \frac{\Delta(u)}{u^{1 / \beta}} S\right]$ and $I_{k, l}(u)=D_{k}(u) \times F_{l}(u)$ with $S>0$. Moreover, let $N_{S, u}=\left[\frac{u^{1 / \beta} \ln m(u)}{m(u) \Delta(u) S}\right]$ and $m_{k, l}^{-\epsilon}(u)=m(u)\left(1+(b-\epsilon)\left((l-k) \frac{\Delta(u)}{u^{1 / \beta}} S\right)^{2}\right)$ with $b=\frac{B}{2 A}$. 
Proof of case $\lim _{u \rightarrow \infty} \frac{T_{u}}{\Delta(u)}=\infty$.

Upper bound of $\pi_{T_{u}}(u)$. Clearly, we have

$$
\begin{aligned}
\pi_{T_{u}}(u) & \leq \sum_{k=0}^{\left[\frac{T_{u}}{\Delta(u) S}\right]+1} \sum_{l=-N_{S, u}-1+k}^{N_{S, u}+2+k} \mathbb{P}\left(\sup _{(t, s) \in I_{k, l}(u)} Z_{u}(s, t)>m(u)\right) \\
& \leq \sum_{k=0}^{\left[\frac{T_{u}}{\Delta(u) S}\right]+1} \sum_{l=-N_{S, u}-1+k}^{N_{S, u}+2+k} \mathbb{P}\left(\sup _{(t, s) \in I_{k, l}(u)} \overline{Z_{u}}(s, t)>m_{k, l}^{-\epsilon}(u)\right),
\end{aligned}
$$

In order to apply Lemma 5.1, we have to check conditions P1-P4, for appropriately chosen $K_{u}, g_{k, l}, \theta_{k, l}$. Let

$$
X^{(u, k, l)}(t, s):=\overline{Z_{u}}\left(\tau_{u}+l \frac{\Delta(u)}{u^{1 / \beta}} S+\frac{\Delta(u)}{u^{1 / \beta}} s, k \frac{\Delta(u)}{u^{1 / \beta}} S+\frac{\Delta(u)}{u^{1 / \beta}} t\right)
$$

with $(s, t) \in[0, S]^{2}$ and $(k, l) \in K_{u}:=\left\{(k, l), 0 \leq k \leq\left[\frac{T_{u}}{\Delta(u) S}\right]+1,-N_{S, u}-1+k \leq l \leq N_{S, u}+2+k\right\}$. Then, let $g_{k, l}(u):=m_{k, l}^{-\epsilon}(u)$ and comparing (10) in Lemma 5.1 and (13) in Lemma 5.4, we introduce

$$
\theta_{k, l}\left(u, t, s, t_{1}, s_{1}\right):=\frac{\sigma^{2}\left(\Delta(u)\left|s-s_{1}\right|\right)+\sigma^{2}\left(\Delta(u)\left|t-t_{1}\right|\right)}{\sigma^{2}(\Delta(u))} \frac{\sigma^{2}(\Delta(u))}{2 \sigma^{2}\left(u^{1 / \beta} \tau^{*}\right)}\left(m_{k, l}^{-\epsilon}(u)\right)^{2},
$$

for $(t, s),\left(t_{1}, s_{1}\right) \in[0, S]^{2},(k, l) \in K_{u}$.

Assumption $\mathbf{P 1}$ holds straightforwardly. In order to show P2, we observe that, by definition of $\Delta(u)$,

$$
\lim _{u \rightarrow \infty} \sup _{(k, l) \in K_{u}}\left|\frac{\sigma^{2}(\Delta(u))}{2 \sigma^{2}\left(u^{1 / \beta} \tau^{*}\right)}\left(m_{k, l}^{-\epsilon}(u)\right)^{2}-1\right|=0
$$

and, by the UCT,

$$
\lim _{u \rightarrow \infty}\left|\frac{\sigma^{2}\left(\Delta(u)\left|s-s_{1}\right|\right)+\sigma^{2}\left(\Delta(u)\left|t-t_{1}\right|\right)}{\sigma^{2}(\Delta(u))}-\right| s-\left.s_{1}\right|^{2 \alpha_{0}}-\left|t-t_{1}\right|^{2 \alpha_{0}} \mid=0, \quad(t, s),\left(t_{1}, s_{1}\right) \in[0, S]^{2} .
$$

Therefore using UCT again, we conclude that

$$
\begin{aligned}
& \lim _{u \rightarrow \infty} \sup _{(k, l) \in K_{u}}\left|\theta_{k, l}\left(u, t, s, t_{1}, s_{1}\right)-\right| s-\left.s_{1}\right|^{2 \alpha_{0}}-\left|t-t_{1}\right|^{2 \alpha_{0}} \mid \\
\leq & \lim _{u \rightarrow \infty}\left|\frac{\sigma^{2}\left(\Delta(u)\left|s-s_{1}\right|\right)+\sigma^{2}\left(\Delta(u)\left|t-t_{1}\right|\right)}{\sigma^{2}(\Delta(u))}-\right| s-\left.s_{1}\right|^{2 \alpha_{0}}-\left|t-t_{1}\right|^{2 \alpha_{0}} \mid \\
& +\lim _{u \rightarrow \infty} \sup _{(k, l) \in K_{u}}\left|\frac{\sigma^{2}(\Delta(u))}{2 \sigma^{2}\left(u^{1 / \beta} \tau^{*}\right)}\left(m_{k, l}^{-\epsilon}(u)\right)^{2}-1\right| \frac{\sigma^{2}\left(\Delta(u)\left|s-s_{1}\right|\right)+\sigma^{2}\left(\Delta(u)\left|t-t_{1}\right|\right)}{\sigma^{2}(\Delta(u))} \\
\leq & \lim _{u \rightarrow \infty}\left|\frac{\sigma^{2}\left(\Delta(u)\left|s-s_{1}\right|\right)+\sigma^{2}\left(\Delta(u)\left|t-t_{1}\right|\right)}{\sigma^{2}(\Delta(u))}-\right| s-\left.s_{1}\right|^{2 \alpha_{0}}-\left|t-t_{1}\right|^{2 \alpha_{0}} \mid \\
& +\lim _{u \rightarrow \infty} \sup _{(k, l) \in K_{u}} \mathbb{Q} S^{2 \alpha_{0}}\left|\frac{\sigma^{2}(\Delta(u))}{2 \sigma^{2}\left(u^{1 / \beta} \tau^{*}\right)}\left(m_{k, l}^{-\epsilon}(u)\right)^{2}-1\right| \\
\rightarrow & 0, \quad(t, s),\left(t_{1}, s_{1}\right) \in[0, S]^{2},
\end{aligned}
$$

which implies that $\mathbf{P 2}$ is satisfied.

In order to check P3, we have that by UCT, with noting that $g_{1}(t)$ defined in (15) is regularly varying at 0 with index $2 \alpha_{0}-\gamma_{1}$,

$$
\varlimsup_{u \rightarrow \infty} \sup _{(k, l) \in K_{u}} \sup _{(t, s) \neq\left(t_{1}, s_{1}\right) \in[0, S]^{2}} \frac{\theta_{k, l}\left(u, t, s, t_{1}, s_{1}\right)}{\left|s-s_{1}\right|^{\gamma_{1}}+\left|t-t_{1}\right|^{\gamma_{1}}}
$$




$$
\begin{aligned}
& \leq \varlimsup_{u \rightarrow \infty} \sup _{(k, l) \in K_{u}} \sup _{(t, s) \neq\left(t_{1}, s_{1}\right) \in[0, S]^{2}} 2 \frac{\sigma^{2}\left(\Delta(u)\left|s-s_{1}\right|\right)+\sigma^{2}\left(\Delta(u)\left|t-t_{1}\right|\right)}{\sigma^{2}(\Delta(u))\left(\left|s-s_{1}\right|^{\gamma_{1}}+\left|t-t_{1}\right|^{\gamma_{1}}\right)} \\
& \leq 2 \overline{\lim }_{u \rightarrow \infty} \sup _{(t, s) \neq\left(t_{1}, s_{1}\right) \in[0, S]^{2}} \frac{\sigma^{2}\left(\Delta(u)\left|s-s_{1}\right|\right)}{\sigma^{2}(\Delta(u))\left|s-s_{1}\right|^{\gamma_{1}}}+2 \overline{\lim }_{u \rightarrow \infty} \sup _{(t, s) \neq\left(t_{1}, s_{1}\right) \in[0, S]^{2}} \frac{\sigma^{2}\left(\Delta(u)\left|t-t_{1}\right|\right)}{\sigma^{2}(\Delta(u))\left|t-t_{1}\right|^{\gamma_{1}}} \\
& =4 \varlimsup_{\lim _{u \rightarrow \infty}} \sup _{t \in[0, S]} \frac{g_{1}(\Delta(u) t)}{g_{1}(\Delta(u))} \leq 8 S^{2 \alpha_{0}-\gamma_{1}}<\infty
\end{aligned}
$$

holds with $0<\gamma_{1}<2 \alpha_{0}$. Next we focus on P4. First, in light of UCT and (24)

$$
\begin{aligned}
& \lim _{\epsilon \rightarrow 0} \varlimsup_{u \rightarrow \infty} \sup _{(k, l) \in K_{u}\left|(t, s)-\left(t_{1}, s_{1}\right)\right|<\epsilon,(t, s),\left(t_{1}, s_{1}\right) \in[0, S]^{2}}\left|\theta_{k, l}(u, t, s, 0,0)-\theta_{k, l}\left(u, t_{1}, s_{1}, 0,0\right)\right| \\
\leq & 2 \lim _{\epsilon \rightarrow 0} \varlimsup_{u \rightarrow \infty} \sup _{s, t, s_{1}, t_{1} \in[0, \epsilon],}\left|\frac{\sigma^{2}(\Delta(u) s)+\sigma^{2}(\Delta(u) t)-\sigma^{2}\left(\Delta(u) s_{1}\right)-\sigma^{2}\left(\Delta(u) t_{1}\right)}{\sigma^{2}(\Delta(u))}\right|=0 .
\end{aligned}
$$

Second, it follows from Lemma 5.4 and and UCT that

$$
\begin{aligned}
& \left|\left(m_{k, l}^{-\epsilon}(u)\right)^{2}\left(1-r_{u}\left(s_{l}(u)+\frac{\Delta(u)}{u^{1 / \beta}} s, t_{k}(u)+\frac{\Delta(u)}{u^{1 / \beta}} t, s_{l}(u), t_{k}(u)\right)\right)-\theta_{k, l}(u, t, s, 0,0)\right| \\
\leq & \left|\left(m_{k, l}^{-\epsilon}(u)\right)^{2} \frac{1-r_{u}\left(s_{l}(u)+\frac{\Delta(u)}{u^{1 / \beta}} s, t_{k}(u)+\frac{\Delta(u)}{u^{1 / \beta}} t, s_{l}(u), t_{k}(u)\right)}{\theta_{k, l}(u, t, s, 0,0)}-1\right| \theta_{k, l}(u, t, s, 0,0) \\
\leq & \mathbb{Q}_{0} S^{2 \alpha_{0}}\left|\left(m_{k, l}^{-\epsilon}(u)\right)^{2} \frac{1-r_{u}\left(s_{l}(u)+\frac{\Delta(u)}{u^{1 / \beta}} s, t_{k}(u)+\frac{\Delta(u)}{u^{1 / \beta}} t, s_{l}(u), t_{k}(u)\right)}{\theta_{k, l}(u, t, s, 0,0)}-1\right| \Rightarrow 0, \quad u \rightarrow \infty,
\end{aligned}
$$

with respect to $(k, l) \in K_{u}$ and $(t, s) \in[0, S]^{2}$, where $s_{l}(u)=\tau_{u}+l \frac{\Delta(u)}{u^{1 / \beta}} S$ and $t_{k}(u)=k \frac{\Delta(u)}{u^{1 / \beta}} S$.

The above leads to, for $\left|(t, s)-\left(t_{1}, s_{1}\right)\right|<\epsilon$,

$$
\begin{aligned}
& \left(m_{k, l}^{-\epsilon}(u)\right)^{2} \mathbb{E}\left(\left(X^{(u, k, l)}(s, t)-X^{(u, k, l)}\left(s_{1}, t_{1}\right)\right) X^{(u, k, l)}(0,0)\right) \\
\leq & \left|\left(m_{k, l}^{-\epsilon}(u)\right)^{2}\left(1-r_{u}\left(s_{l}(u)+\frac{\Delta(u)}{u^{1 / \beta}} s, t_{k}(u)+\frac{\Delta(u)}{u^{1 / \beta}} t, s_{l}(u), t_{k}(u)\right)\right)-\theta_{k, l}(u, t, s, 0,0)\right| \\
& +\left|\left(m_{k, l}^{-\epsilon}(u)\right)^{2}\left(1-r_{u}\left(s_{l}(u)+\frac{\Delta(u)}{u^{1 / \beta}} s_{1}, t_{k}(u)+\frac{\Delta(u)}{u^{1 / \beta}} t_{1}, s_{l}(u), t_{k}(u)\right)\right)-\theta_{k, l}\left(u, t_{1}, s_{1}, 0,0\right)\right| \\
& +\left|\theta_{k, l}(u, t, s, 0,0)-\theta_{k, l}\left(u, t_{1}, s_{1}, 0,0\right)\right| \Rightarrow 0, \quad u \rightarrow \infty, \epsilon \rightarrow 0 .
\end{aligned}
$$

with respect to $(k, l) \in K_{u},(t, s),\left(t_{1}, s_{1}\right) \in[0, S]^{2}$, which confirms that $\mathbf{P} 4$ is fulfilled.

Thus in view of Lemma 5.1

$$
\frac{\mathbb{P}\left(\sup _{(t, s) \in I_{k, l}(u)} \overline{Z_{u}}(s, t)>m_{k, l}^{-\epsilon}(u)\right)}{\Psi\left(m_{k, l}^{-\epsilon}(u)\right)} \rightarrow \mathcal{H}_{V}\left([0, S]^{2}\right)=\left(\mathcal{H}_{B_{\alpha_{0}}}[0, S]\right)^{2}, \quad u \rightarrow \infty,
$$

where $V(t, s)=B_{\alpha_{0}}^{(1)}(t)+B_{\alpha_{0}}^{(2)}(s)$, with $B_{\alpha_{0}}^{(1)}$ and $B_{\alpha_{0}}^{(2)}$ being two independent fBms with index $\alpha_{0}$. Then, continuing (22), in view of (11) we have

$$
\begin{aligned}
\pi_{T_{u}}(u) & \leq \sum_{k=0}^{\left[\frac{T_{u}}{\Delta(u) S}\right]+1} \sum_{l=-N_{S, u}-1+k}^{N_{S, u}+2+k}\left(\mathcal{H}_{B_{\alpha_{0}}}[0, S]\right)^{2} \Psi\left(m_{k, l}^{-\epsilon}(u)\right)(1+o(1)) \\
& \leq \sum_{k=0}^{\left[\frac{T_{u}}{\Delta(u) S}\right]+1}\left(\mathcal{H}_{B_{\alpha_{0}}}[0, S]\right)^{2} \Psi(m(u)) \sum_{l=-N_{S, u}-2}^{N_{S, u}+3} e^{-(b-\epsilon)\left(\operatorname{lm}(u) \frac{\Delta(u)}{u^{1 / \beta} S}\right)^{2}}(1+o(1))
\end{aligned}
$$




$$
\begin{aligned}
& \leq\left(\frac{\mathcal{H}_{B_{\alpha_{0}}}[0, S]}{S}\right)^{2}(b-\epsilon)^{-1 / 2}\left[\frac{T_{u}}{\Delta(u)}\right] \frac{u^{1 / \beta}}{m(u) \Delta(u)} \Psi(m(u)) \int_{-\infty}^{\infty} e^{-x^{2}} d x(1+o(1)) \\
& \sim\left(\mathcal{H}_{B_{\alpha_{0}}}\right)^{2}(b-\epsilon)^{-1 / 2} \sqrt{\pi} \frac{u^{1 / \beta} T_{u}}{(\Delta(u))^{2} m(u)} \Psi(m(u)), \text { as } u \rightarrow \infty,
\end{aligned}
$$

with $b=\frac{B}{2 A}$ (see Lemma 5.3). Hence, letting $\epsilon \rightarrow 0$, we obtain the upper bound for $\pi_{T_{u}}(u$ ).

Lower bound of $\pi_{T_{u}}(u)$. Set

$\Gamma_{\delta, 1}=\left\{\left(k, l, k_{1}, l_{1}\right): 0 \leq k \leq k_{1} \leq\left[\frac{T_{u}}{\Delta(u) S}\right],\left|k_{1}-k\right| \leq \frac{\delta u^{1 / \beta}}{\Delta(u) S},-N_{S, u}+k \leq l \leq l_{1} \leq N_{S, u}+k, I_{k, l}(u) \cap I_{k_{1}, l_{1}}(u)=\emptyset\right\}$,

$\Gamma_{\delta, 2}=\left\{\left(k, l, k_{1}, l_{1}\right): 0 \leq k \leq k_{1} \leq\left[\frac{T_{u}}{\Delta(u) S}\right],\left|k_{1}-k\right| \leq \frac{\delta u^{1 / \beta}}{\Delta(u) S},-N_{S, u}+k \leq l \leq l_{1} \leq N_{S, u}+k, I_{k, l}(u) \cap I_{k_{1}, l_{1}}(u) \neq \emptyset\right\}$,

$\Gamma_{\delta, 3}=\left\{\left(k, l,, k_{1}, l_{1}\right): 0 \leq k \leq k_{1} \leq\left[\frac{T_{u}}{\Delta(u) S}\right], \frac{\delta u^{1 / \beta}}{\Delta(u) S}<\left|k_{1}-k\right| \leq \frac{u^{1 / \beta}}{\Delta(u) S} e^{\frac{1-a_{\delta}}{4+4 a_{\delta}} m^{2}(u)},-N_{S, u}+k \leq l \leq l_{1} \leq N_{S, u}+k\right\}$,

$\Gamma_{\delta, 4}=\left\{\left(k, l, k_{1}, l_{1}\right): 0 \leq k \leq k_{1} \leq\left[\frac{T_{u}}{\Delta(u) S}\right],\left|k_{1}-k\right|>\frac{u^{1 / \beta}}{\Delta(u) S} e^{\frac{1-a_{\delta}}{4+4 a_{\delta}} m^{2}(u)},-N_{S, u}+k \leq l \leq l_{1} \leq N_{S, u}+k\right\}$ with $a_{\delta}$

defined in Lemma 5.5. We have

$$
\pi_{T_{u}}(u) \geq \sum_{k=0}^{\left[\frac{T_{u}}{\Delta(u) S}\right]} \sum_{l=-N_{S, u}+k}^{N_{S, u}+k} \mathbb{P}\left(\sup _{(t, s) \in I_{k, l}(u)} Z_{u}(s, t)>m(u)\right)-\left(\Sigma_{1}(u)+\Sigma_{2}(u)+\Sigma_{3}(u)+\Sigma_{4}(u)\right),
$$

where

$$
\Sigma_{i}(u)=\sum_{\left(k, l, k_{1}, l_{1}\right) \in \Gamma_{\delta, i}} \mathbb{P}\left(\sup _{(t, s) \in I_{k, l}(u)} Z_{u}(s, t)>m(u) \sup _{\left(t_{1}, s_{1}\right) \in I_{k_{1}, l_{1}}(u)} Z_{u}\left(s_{1}, t_{1}\right)>m(u)\right), \quad i=1,2,3,4 .
$$

The same lines of reasoning, as presented in the proof of the upper bound of $\pi_{T_{u}}(u)$, give the lower bound for $\sum_{k=0}^{\left[\frac{T_{u}}{\Delta(u) S}\right]} \sum_{l=-N_{S, u}+k}^{N_{S, u}+k} \mathbb{P}\left(\sup _{(t, s) \in I_{k, l}(u)} Z_{u}(s, t)>m(u)\right)$, which asymptotically agrees with the upper bound. Thus the remaining task is to prove that $\Sigma_{i}(u), i=1,2,3,4$ are asymptotically negligible.

Upper bound of $\Sigma_{1}(u)$. In light of Lemma 5.4, there exists a positive constant $\delta>0$ such that for $u$ large enough, all $\left(t, s, t_{1}, s_{1}\right) \in I_{k, l}(u) \times I_{k_{1}, l_{1}}(u)$ with $\left(k, l, k_{1}, l_{1}\right) \in \Gamma_{\delta, 1}$,

$$
1 / 2<\frac{1-r_{u}\left(s, t, s_{1}, t_{1}\right)}{\frac{\sigma^{2}\left(u^{1 / \beta}\left|s-s_{1}\right|\right)+\sigma^{2}\left(u^{1 / \beta}\left|t-t_{1}\right|\right)}{2 \sigma^{2}\left(u^{1 / \beta} \tau^{*}\right)}}<2 .
$$

Moreover, by UCT, we have

$$
\begin{aligned}
2 \leq \mathbb{E}\left(\overline{Z_{u}}(s, t)+\overline{Z_{u}}\left(s_{1}, t_{1}\right)\right)^{2} & =4-2\left(1-r_{u}\left(s, t, s_{1}, t_{1}\right)\right) \\
& \leq 4-\frac{\sigma^{2}\left(u^{1 / \beta}\left|s-s_{1}\right|\right)+\sigma^{2}\left(u^{1 / \beta}\left|t-t_{1}\right|\right)}{2 \sigma^{2}\left(u^{1 / \beta} \tau^{*}\right)} \\
& \leq 4-\mathbb{Q}_{2} \frac{\left|l_{1}-l\right|^{\gamma_{1}} S^{\gamma_{1}}+\left|k_{1}-k\right|^{\gamma_{1}} S^{\gamma_{1}}}{m^{2}(u)},
\end{aligned}
$$

where $0<\gamma_{1}<\min \left(2 \alpha_{\infty}, \gamma\right)$.

Thus

$$
\begin{aligned}
\Sigma_{1}(u) & \leq \sum_{\left(k, l, k_{1}, l_{1}\right) \in \Gamma_{\delta, 1}} \mathbb{P}\left(\sup _{(t, s) \in I_{k, l}(u)} \overline{Z_{u}}(s, t)>m_{k, l}^{-\epsilon}(u), \sup _{\left(t_{1}, s_{1}\right) \in I_{k_{1}, l_{1}}(u)} \overline{Z_{u}}\left(s_{1}, t_{1}\right)>m_{k_{1}, l_{1}}^{-\epsilon}(u)\right) \\
& \leq \sum_{\left(k, l, k_{1}, l_{1}\right) \in \Gamma_{\delta, 1}} \mathbb{P}\left(\sup _{\left(t, s, t_{1}, s_{1}\right) \in I_{k, l}(u) \times I_{k_{1}, l_{1}}(u)} \overline{Z_{u}}(s, t)+\overline{Z_{u}}\left(s_{1}, t_{1}\right)>2 \hat{m}_{k, l, k_{1}, l_{1}}^{-\epsilon}(u)\right)
\end{aligned}
$$




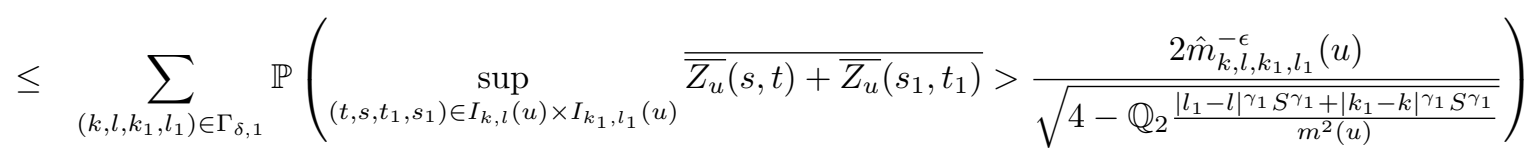

with $\hat{m}_{k, l, k_{1}, l_{1}}^{-\epsilon}(u)=\min \left(m_{k, l}^{-\epsilon}(u), m_{k_{1}, l_{1}}^{-\epsilon}(u)\right)$.

In order to bound the above sum, we introduce

$$
r_{u}\left(t, s, t_{1}, s_{1}, t^{\prime}, s^{\prime}, t_{1}^{\prime}, s_{1}^{\prime}\right):=\mathbb{E}\left(\left(\overline{\overline{Z_{u}}(s, t)+\overline{Z_{u}}\left(s_{1}, t_{1}\right)}\right)\left(\overline{\overline{Z_{u}}}\left(s^{\prime}, t^{\prime}\right)+\overline{Z_{u}}\left(s_{1}^{\prime}, t_{1}^{\prime}\right)\right)\right)
$$

and observe that for $\left(t, s, t_{1}, s_{1}\right),\left(t^{\prime}, s^{\prime}, t_{1}^{\prime}, s_{1}^{\prime}\right) \in I_{k, l}(u) \times I_{k_{1}, l_{1}}(u)$,

$$
\begin{aligned}
1-r_{u}\left(t, s, t_{1}, s_{1}, t^{\prime}, s^{\prime}, t_{1}^{\prime}, s_{1}^{\prime}\right) & \leq \frac{\mathbb{E}\left(\overline{Z_{u}}(s, t)+\overline{Z_{u}}\left(s_{1}, t_{1}\right)-\overline{Z_{u}}\left(s^{\prime}, t^{\prime}\right)-\overline{Z_{u}}\left(s_{1}^{\prime}, t_{1}^{\prime}\right)\right)^{2}}{2 \sqrt{\mathbb{E}\left(\overline{Z_{u}}(s, t)+\overline{Z_{u}}\left(s_{1}, t_{1}\right)\right)^{2}} \sqrt{\mathbb{E}\left(\overline{Z_{u}}\left(s^{\prime}, t^{\prime}\right)+\overline{Z_{u}}\left(s_{1}^{\prime}, t_{1}^{\prime}\right)\right)^{2}}} \\
& \leq \frac{\mathbb{E}\left(\overline{Z_{u}}(s, t)-\overline{Z_{u}}\left(s^{\prime}, t^{\prime}\right)\right)^{2}+\mathbb{E}\left(\overline{Z_{u}}\left(s_{1}, t_{1}\right)-\overline{Z_{u}}\left(s_{1}^{\prime}, t_{1}^{\prime}\right)\right)^{2}}{2} \\
& \leq 1-r_{u}\left(s, t, s^{\prime}, t^{\prime}\right)+1-r_{u}\left(s_{1}, t_{1}, s_{1}^{\prime}, t_{1}^{\prime}\right) \\
& \leq \frac{\sigma^{2}\left(u^{1 / \beta}\left|s-s^{\prime}\right|\right)+\sigma^{2}\left(u^{1 / \beta}\left|t-t^{\prime}\right|\right)}{\sigma^{2}\left(u^{1 / \beta} \tau^{*}\right)}+\frac{\sigma^{2}\left(u^{1 / \beta}\left|s_{1}-s_{1}^{\prime}\right|\right)+\sigma^{2}\left(u^{1 / \beta}\left|t_{1}-t_{1}^{\prime}\right|\right)}{\sigma^{2}\left(u^{1 / \beta} \tau^{*}\right)} \\
& \leq \mathbb{Q}_{3} S^{2} \frac{\left(\frac{u^{1 / \beta}}{\Delta(u)}\right)^{\gamma_{2}}\left(\left|s-s^{\prime}\right|^{\gamma_{2}}+\left|t-t^{\prime}\right| \gamma_{2}+\left|s_{1}-s_{1}^{\prime}\right|^{\gamma_{2}}+\left|t_{1}-t_{1}^{\prime}\right|^{\gamma_{2}}\right)}{m^{2}(u)},
\end{aligned}
$$

with $0<\gamma_{2}<\min \left(2 \alpha_{\infty}, \gamma\right)$ and $S \geq 1$.

Next we define a centered homogenous Gaussian field $\left\{X_{u}^{*}\left(s, t, s_{1}, t_{1}\right),\left(s, t, s_{1}, t_{1}\right) \in \mathbb{R}^{4}\right\}$ so that $X_{u}^{*}\left(s, t, s_{1}, t_{1}\right):=$ $\left(X_{u}^{1}(s)+X_{u}^{2}(t)+X_{u}^{3}\left(s_{1}\right)+X_{u}^{4}\left(t_{1}\right)\right) / 2$ with $X_{u}^{i}(s), 1 \leq i \leq 4$, being i.i.d. centered stationary Gaussian processes with covariance function

$$
r_{u}\left(s, s^{\prime}\right)=\exp \left(-8 \mathbb{Q}_{3} S^{2}\left(\frac{u^{1 / \beta}}{\Delta(u)}\right)^{\gamma_{2}} \frac{1}{m^{2}(u)}\left|s-s^{\prime}\right|^{\gamma_{2}}\right) .
$$

Let $r_{u}^{*}\left(s, t, s_{1}, t_{1}, s^{\prime}, t^{\prime}, s_{1}^{\prime}, t_{1}^{\prime}\right)$ be the covariance function of $X_{u}^{*}\left(s, t, s_{1}, t_{1}\right)$. It is straightforward to check that for $\left(t, s, t_{1}, s_{1}\right),\left(t^{\prime}, s^{\prime}, t_{1}^{\prime}, s_{1}^{\prime}\right) \in I_{k, l}(u) \times I_{k_{1}, l_{1}}(u)$,

$$
r_{u}\left(s, t, s_{1}, t_{1}, s^{\prime}, t^{\prime}, s_{1}^{\prime}, t_{1}^{\prime}\right) \geq r_{u}^{*}\left(s, t, s_{1}, t_{1}, s^{\prime}, t^{\prime}, s_{1}^{\prime}, t_{1}^{\prime}\right) .
$$

In light of Slepian's inequality ( see, e.g., [1] or [13]) and Lemma 6.1 in [13], we have

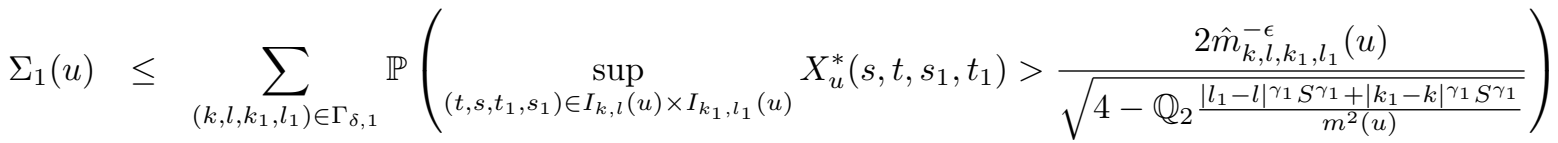

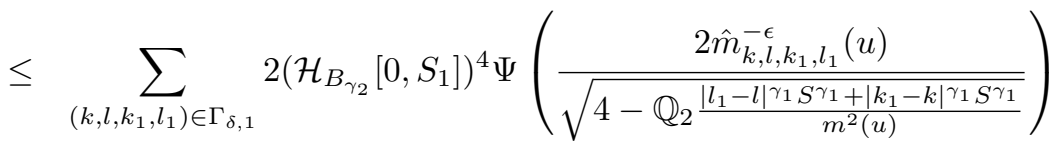

$$
\begin{aligned}
& \leq \sum_{\left(k, l, k_{1}, l_{1}\right) \in \Gamma_{\delta, 1}} 4\left(\mathcal{H}_{B_{\gamma_{2}}}\left[0, S_{1}\right]\right)^{4} \Psi\left(\hat{m}_{k, l, k_{1}, l_{1}}^{-\epsilon}(u)\right) e^{-\mathbb{Q}_{4}\left(\left|l_{1}-l\right|^{\gamma_{1}} S^{\gamma_{1}}+\left|k_{1}-k\right|^{\gamma_{1}} S^{\gamma_{1}}\right)} \\
& \leq \sum_{k=0}^{\left[\frac{T_{u}}{\Delta(u) S}\right]} \sum_{l=-N_{S, u}+k}^{N_{S, u}+k} 4\left(\frac{\mathcal{H}_{B_{\gamma_{2}}}\left[0, S_{1}\right]}{S_{1}}\right)^{4} \Psi\left(m_{k, l}^{-\epsilon}(u)\right) S_{1}^{4} \sum_{i \geq 0, j \geq 0, i+j \geq 1} e^{-\mathbb{Q}_{4}\left(i^{\gamma_{1}} S^{\gamma_{1}}+j^{\gamma_{1}} S^{\gamma_{1}}\right)}
\end{aligned}
$$




$$
\leq 8\left(\frac{\mathcal{H}_{B_{\gamma_{2}}}\left[0, S_{1}\right]}{S_{1}}\right)^{4}(b-\epsilon)^{-1 / 2} \sqrt{\pi} \frac{u^{1 / \beta} T_{u}}{(\Delta(u))^{2} m(u)} \Psi(m(u)) S_{1}^{4} e^{-\mathbb{Q}_{5} S^{\gamma_{1}}}
$$

with $S_{1}=\left(2 \mathbb{Q}_{3}\right)^{2 / \gamma_{2}} S^{1+4 / \gamma_{2}}$. Letting $S \rightarrow \infty$, we get that $\Sigma_{1}(u)=o\left(\pi_{T_{u}}(u)\right)$ as $u \rightarrow \infty$.

Upper bound of $\Sigma_{2}(u)$. We have

$$
\Sigma_{2}(u) \leq \sum_{\left(k, l, k_{1}, l_{1}\right) \in \Gamma_{\delta, 2}} p_{k, l, k_{1}, l_{1}}^{(1)}(u)+\sum_{\left(k, l, k_{1}, l_{1}\right) \in \Gamma_{\delta, 2}} p_{k, l, k_{1}, l_{1}}^{(2)}(u)
$$

with

$$
\begin{aligned}
& p_{k, l, k_{1}, l_{1}}^{(1)}(u):=\mathbb{P}\left(\sup _{(t, s) \in I_{k, l}(u)} Z_{u}(s, t)>m(u) \sup _{\left(t_{1}, s_{1}\right) \in I_{k_{1}, l_{1}}^{1}(u)} Z_{u}\left(s_{1}, t_{1}\right)>m(u)\right) \\
& p_{k, l, k_{1}, l_{1}}^{(2)}(u):=\mathbb{P}\left(\sup _{(t, s) \in I_{k, l}(u)} Z_{u}(s, t)>m(u) \sup _{\left(t_{1}, s_{1}\right) \in I_{k_{1}, l_{1}}^{2}(u)} Z_{u}\left(s_{1}, t_{1}\right)>m(u)\right),
\end{aligned}
$$

where, without loss of generality we assume that $k+1=k_{1}$ and, $l=l_{1}$ or $l \pm 1=l_{1}$ and

$$
\begin{aligned}
& I_{k_{1}, l_{1}}^{1}(u)=\left[(k+1) \frac{\Delta(u)}{u^{1 / \beta}} S,(k+1) \frac{\Delta(u)}{u^{1 / \beta}} S+\frac{\Delta(u)}{u^{1 / \beta}} \sqrt{S}\right] \times F_{l_{1}}(u), \\
& I_{k_{1}, l_{1}}^{2}(u)=\left[(k+1) \frac{\Delta(u)}{u^{1 / \beta}} S+\frac{\Delta(u)}{u^{1 / \beta}} \sqrt{S},(k+2) \frac{\Delta(u)}{u^{1 / \beta}} S,\right] \times F_{l_{1}}(u) .
\end{aligned}
$$

Following the same argument as given in the proof of $\Sigma_{1}(u)$, we get

$$
\begin{aligned}
\sum_{\left(k, l, k_{1}, l_{1}\right) \in \Gamma_{\delta, 2}} p_{k, l, k_{1}, l_{1}}^{(1)}(u) & \leq \sum_{k_{1}=0}^{\left[\frac{T_{u}}{\Delta(u) S}\right]+1} \sum_{l_{1}=-N_{S, u}-1+k}^{N_{S, u}+2+k} \mathbb{P}\left(\sup _{\left(t_{1}, s_{1}\right) \in I_{k_{1}, l_{1}}^{1}(u)} Z_{u}\left(s_{1}, t_{1}\right)>m(u)\right) \\
& \leq \sum_{k=0}^{\left[\frac{T_{u}}{\Delta(u) S}\right]+1} \sum_{l=-N_{S, u}-1+k}^{N_{S, u}+2+k} \mathcal{H}_{B_{\alpha_{0}}}[0, \sqrt{S}] \mathcal{H}_{B_{\alpha_{0}}}[0, S] \Psi\left(m_{k, l}^{-\epsilon}(u)\right)(1+o(1)) \\
& \leq \frac{2}{\sqrt{S}} \frac{\mathcal{H}_{B_{\alpha_{0}}}[0, \sqrt{S}]}{\sqrt{S}} \frac{\mathcal{H}_{B_{\alpha_{0}}}[0, S]}{S}(b-\epsilon)^{-1 / 2} \sqrt{\pi} \frac{u^{1 / \beta} T_{u}}{(\Delta(u))^{2} m(u)} \Psi(m(u)) .
\end{aligned}
$$

and, with the same $S_{1}$ as above,

$$
\begin{aligned}
\sum_{\left(k, l, k_{1}, l_{1}\right) \in \Gamma_{\delta, 2}} p_{k, l, k_{1}, l_{1}}^{(2)}(u) & \leq \sum_{\left(k, l, k_{1}, l_{1}\right) \in \Gamma_{\delta, 2}} \mathbb{P}\left(\sup _{\left(t, s, t_{1}, s_{1}\right) \in I_{k, l}(u) \times I_{k_{1}, l_{1}(u)}} \overline{\overline{Z_{u}}(s, t)+\overline{Z_{u}}\left(s_{1}, t_{1}\right)}>\frac{2 \hat{m}_{k, l, k_{1}, l_{1}}^{-\epsilon}(u)}{\sqrt{4-\mathbb{Q}_{3} \frac{S^{\gamma_{1} / 2}}{m^{2}(u)}}}\right) \\
& \leq \sum_{\left(k, l, k_{1}, l_{1}\right) \in \Gamma_{\delta, 2}} 2\left(\mathcal{H}_{B_{\alpha_{0}}}\left[0, S_{1}\right]\right)^{4} \Psi\left(\frac{2 \hat{m}_{k, l, k_{1}, l_{1}}^{-\epsilon}(u)}{\sqrt{4-\mathbb{Q}_{3} \frac{S^{\gamma_{1} / 2}}{m^{2}(u)}}}\right) \\
& \leq \sum_{k=0}^{\left[\frac{T_{u}}{\Delta(u) S}\right]} \sum_{l=-N_{S, u}+k}^{N_{S, u}+k} 4\left(\frac{\mathcal{H}_{B_{\alpha_{0}}}\left[0, S_{1}\right]}{S_{1}}\right)^{4} \Psi\left(m_{k, l}^{-\epsilon}(u)\right) S_{1}^{4} e^{-\mathbb{Q}_{3} S^{\gamma_{1} / 2}} \\
& \leq 8\left(\frac{\mathcal{H}_{B_{\alpha_{0}}}\left[0, S_{1}\right]}{S_{1}}\right)^{4}(b-\epsilon)^{-1 / 2} \sqrt{\pi} \frac{u^{1 / \beta} T_{u}}{(\Delta(u))^{2} m(u)} \Psi(m(u)) S_{1}^{4} e^{-\mathbb{Q}_{3} S^{\gamma_{1} / 2}} .
\end{aligned}
$$

Combination of (27) with (28) implies that $\Sigma_{2}(u)=o\left(\pi_{T_{u}}(u)\right)$ as $u \rightarrow \infty$. 
Upper bound of $\Sigma_{3}(u)$. The idea of this part of the proof is to apply Borell inequality. For that, without loss of generality, we fix $S=1$. We observe that (similarly as for $\left.\Sigma_{1}(u)\right)$, for $\left(t, s, t_{1}, s_{1}\right),\left(t^{\prime}, s^{\prime}, t_{1}^{\prime}, s_{1}^{\prime}\right) \in I_{k, l}(u) \times I_{k_{1}, l_{1}}(u)$ with $\left(k, l, k_{1}, l_{1}\right) \in \Gamma_{\delta, 3}$,

$$
\mathbb{E}\left(\overline{Z_{u}}(s, t)+\overline{Z_{u}}\left(s_{1}, t_{1}\right)\right)^{2}=2+2 r_{u}\left(s, t, s_{1}, t_{1}\right) \leq 2+2 a_{\delta}<4
$$

and

$$
\begin{aligned}
& \mathbb{E}\left(\overline{Z_{u}}(s, t)+\overline{Z_{u}}\left(s_{1}, t_{1}\right)-\overline{Z_{u}}\left(s^{\prime}, t^{\prime}\right)-\overline{Z_{u}}\left(s_{1}^{\prime}, t_{1}^{\prime}\right)\right)^{2} \\
\leq & 4\left(1-r_{u}\left(s, t, s^{\prime}, t^{\prime}\right)\right)+4\left(1-r_{u}\left(s_{1}, s_{1}^{\prime}, t_{1}, t_{1}^{\prime}\right)\right) \\
\leq & \mathbb{Q}_{6} \frac{\left(\frac{u^{1 / \beta}}{\Delta(u)}\right)^{\gamma_{2}}\left(\left|s-s^{\prime}\right|^{\gamma_{2}}+\left|t-t^{\prime}\right|^{\gamma_{2}}+\left|s_{1}-s_{1}^{\prime}\right|^{\gamma_{2}}+\left|t_{1}-t_{1}^{\prime}\right|^{\gamma_{2}}\right)}{m^{2}(u)} .
\end{aligned}
$$

Thus, by Fernique inequality,

$$
\mathbb{P}\left(\sup _{\left(t, s, t_{1}, s_{1}\right) \in I_{k, l}(u) \times I_{k_{1}, l_{1}}(u)} \overline{Z_{u}}(s, t)+\overline{Z_{u}}\left(s_{1}, t_{1}\right)>x\right) \leq \frac{1}{2} e^{-\frac{x^{2}}{8}},
$$

for any $\left(k, l, k_{1}, l_{1}\right) \in \Gamma_{\delta, 3}$, any $x>0$ and $u$ large enough. This implies that there exists a common positive constant $a$ such that for any $\left(k, l, k_{1}, l_{1}\right) \in \Gamma_{\delta, 3}$ and $u$ large enough

$$
\mathbb{P}\left(\sup _{\left(t, s, t_{1}, s_{1}\right) \in I_{k, l}(u) \times I_{k_{1}, l_{1}}(u)} \overline{Z_{u}}(s, t)+\overline{Z_{u}}\left(s_{1}, t_{1}\right)>a\right) \leq 1 / 2 .
$$

The above implies that we can apply Borell inequality to the sum below uniformly

$$
\begin{aligned}
\Sigma_{3}(u) & \leq \sum_{\left(k, l, k_{1}, l_{1}\right) \in \Gamma_{\delta, 3}} \mathbb{P}\left(\sup _{(t, s) \in I_{k, l}(u)} \overline{Z_{u}}(s, t)>m(u), \sup _{\left(t_{1}, s_{1}\right) \in I_{k_{1}, l_{1}}(u)} \overline{Z_{u}}\left(s_{1}, t_{1}\right)>m(u)\right) \\
& \leq \sum_{\left(k, l, k_{1}, l_{1}\right) \in \Gamma_{\delta, 3}} \mathbb{P}\left(\sup _{\left(t, s, t_{1}, s_{1}\right) \in I_{k, l}(u) \times I_{k_{1}, l_{1}}(u)} \overline{Z_{u}}(s, t)+\overline{Z_{u}}\left(s_{1}, t_{1}\right)>2 m(u)\right) \\
& \leq \mathbb{Q}_{7}\left(\frac{u^{1 /(2 \beta)}}{\Delta(u)} \frac{u^{1 / \beta} \ln m(u)}{m(u) \Delta(u)}\right)^{2} T_{u} e^{\frac{1-a_{\delta}}{4+4 a_{\delta}} m^{2}(u)} \Psi\left(\frac{2 m(u)-a}{\sqrt{2+2 a_{\delta}}}\right) \\
& \leq \mathbb{Q}_{8}\left(\frac{u^{3 /(2 \beta)} \ln m(u)}{m(u) \Delta^{2}(u)}\right)^{2} T_{u} \Psi(m(u)) e^{-m^{2}(u)\left(\frac{1-a_{\delta}}{4+4 a_{\delta}}\right)+\frac{a m(u)}{1+a_{\delta}}} \\
& =o\left(\frac{u^{1 / \beta} T_{u}}{(\Delta(u))^{2} m(u)} \Psi(m(u))\right), \quad u \rightarrow \infty .
\end{aligned}
$$

This implies that $\Sigma_{3}(u)=o\left(\pi_{T_{u}}(u)\right)$ as $u \rightarrow \infty$.

Upper bound of $\Sigma_{4}(u)$. Let $0<\epsilon<\frac{1-2 \beta_{1}}{1+2 \beta_{1}}$ be given. Then, for $u$ large enough, $r_{u}\left(s, t, s_{1}, t_{1}\right)<\epsilon$ holds for $\left|t-t_{1}\right|>$ $e^{\frac{1-a_{\delta}}{8+8 a_{\delta}} m^{2}(u)}$ and $s-t, s_{1}-t_{1} \in E(u)$. Thus similarly as for $\Sigma_{3}(u)$, we have

$$
\begin{aligned}
\Sigma_{4}(u) & \leq \mathbb{Q}_{9}\left(\frac{T_{u}}{\Delta(u)} \frac{u^{1 / \beta} \ln m(u)}{m(u) \Delta(u)}\right)^{2} \Psi\left(\frac{2 m(u)-a}{\sqrt{2+2 \epsilon}}\right) \\
& \leq \mathbb{Q}_{10}\left(\frac{u^{1 / \beta} \ln m(u)}{m(u) \Delta^{2}(u)}\right)^{2} T_{u} \Psi(m(u)) e^{-\left(\frac{1-\epsilon}{2(1+\epsilon)}-\beta_{1}\right) m^{2}(u)+\frac{a m(u)}{1+\epsilon}} \\
& =o\left(\frac{u^{1 / \beta} T_{u}}{(\Delta(u))^{2} m(u)} \Psi(m(u))\right), \quad u \rightarrow \infty
\end{aligned}
$$


Hence $\Sigma_{3}(u)=o\left(\pi_{T_{u}}(u)\right)$ as $u \rightarrow \infty$.

Note that if for some $T_{u}, \Gamma_{\delta, 3}$ or $\Gamma_{\delta, 4}$ are empty then the above inequalities are still valid. This completes the proof of ii).

Proof of case $\lim _{u \rightarrow \infty} \frac{T_{u}}{\Delta(u)}=\rho \in(0, \infty)$. The proof of this case is similar to the proof of the previous case. Thus we focus on the tiny details that differ from the arguments used in the previous case.

For $I_{0, l}^{( \pm \epsilon)}(u)=\left[0,(\rho \pm \epsilon) \frac{\Delta(u)}{u^{1 / \beta}}\right] \times\left[\tau_{u}+l \frac{\Delta(u)}{u^{1 / \beta}} S, \tau_{u}+(l+1) \frac{\Delta(u)}{u^{1 / \beta}} S\right]$ we have

$$
\begin{aligned}
\pi_{T_{u}}(u) & \leq \sum_{l=-N_{S, u}-1}^{N_{S, u}+1} \mathbb{P}\left(\sup _{(t, s) \in I_{0, l}^{+\epsilon}(u)} Z_{u}(s, t)>m(u)\right) \\
& \leq \sum_{l=-N_{S, u}-1}^{N_{S, u}+1} \mathbb{P}\left(\sup _{(t, s) \in I_{0, l}^{+\epsilon}(u)} \overline{Z_{u}}(s, t)>m_{0, l}^{-\epsilon}(u)\right) \\
& \leq \sum_{l=-N_{S, u}-1}^{N_{S, u}+1} \mathcal{H}_{B_{\alpha_{0}}}[0, \rho+\epsilon] \mathcal{H}_{B_{\alpha_{0}}}[0, S] \Psi\left(m_{0, l}^{-\epsilon}(u)\right)(1+o(1)) \\
& \leq \mathcal{H}_{B_{\alpha_{0}}}[0, \rho+\epsilon] \mathcal{H}_{B_{\alpha_{0}}}[0, S] \Psi(m(u)) \sum_{l=-N_{S, u}-1}^{N_{S, u}+1} e^{-(b-\epsilon)\left(l m(u) \frac{\Delta(u)}{u^{1 / \beta}} S\right)^{2}}(1+o(1)) \\
& \leq \mathcal{H}_{B_{\alpha_{0}}}[0, \rho+\epsilon] \mathcal{H}_{B_{\alpha_{0}}}(b-\epsilon)^{-1 / 2} \frac{u^{1 / \beta}}{\Delta(u) m(u)} \Psi(m(u)) \int_{-\infty}^{\infty} e^{-x^{2}} d x(1+o(1)) \\
& \sim \mathcal{H}_{B_{\alpha_{0}}}[0, \rho+\epsilon] \mathcal{H}_{B_{\alpha_{0}}}(b-\epsilon)^{-1 / 2} \sqrt{\pi} \frac{u^{1 / \beta}}{\Delta(u) m(u)} \Psi(m(u))
\end{aligned}
$$

with $N_{S, u}$ and $m_{k, l}^{-\epsilon}(u)$ defined right before (22). Similarly, we have

$$
\begin{aligned}
\pi_{T_{u}}(u) & \geq \sum_{l=-N_{S, u}}^{N_{S, u}} \mathbb{P}\left(\sup _{(t, s) \in I_{0, l}^{-\epsilon}(u)} Z_{u}(s, t)>m(u)\right)-\sum_{i=1}^{2} \Sigma_{i}^{\prime}(u) \\
& \geq \mathcal{H}_{B_{\alpha_{0}}}[0, \rho-\epsilon] \mathcal{H}_{B_{\alpha_{0}}}(b-\epsilon)^{-1 / 2} \sqrt{\pi} \frac{u^{1 / \beta}}{\Delta(u) m(u)} \Psi(m(u))(1+o(1))-\sum_{i=1}^{2} \Sigma_{i}^{\prime}(u),
\end{aligned}
$$

where

$$
\Sigma_{i}^{\prime}(u)=\sum_{\left(l, l_{1}\right) \in \Gamma_{i}^{\prime}} \mathbb{P}\left(\sup _{(t, s) \in I_{0, l}^{-\epsilon}(u)} Z_{u}(s, t)>m(u) \sup _{\left(t_{1}, s_{1}\right) \in I_{0, l_{1}}^{-\epsilon}(u)} Z_{u}\left(s_{1}, t_{1}\right)>m(u)\right), \quad i=1,2,
$$

with $\Gamma_{1}^{\prime}=\left\{\left(l, l_{1}\right),-N_{S, u} \leq l<l_{1}+1 \leq N_{S, u}\right\}$ and $\Gamma_{2}^{\prime}=\left\{\left(l, l_{1}\right),-N_{S, u} \leq l=l_{1}+1 \leq N_{S, u}\right\}$.

Following the same lines of argument as in (26) (see also (27) or (28)), we get that $\sum_{i=1}^{2} \Sigma_{i}^{\prime}(u)$ is negligible compared with the first term in (30). Hence, comparing (29) with (30) and letting $\epsilon \rightarrow 0$, we obtain that for $\frac{T_{u}}{\Delta(u)} \rightarrow \rho \in(0, \infty)$,

$$
\pi_{T_{u}}(u) \sim \mathcal{H}_{B_{\alpha_{0}}}[0, \rho] \mathcal{H}_{B_{\alpha_{0}}} b^{-1 / 2} \sqrt{\pi} \frac{u^{1 / \beta}}{\Delta(u) m(u)} \Psi(m(u))
$$

Finally let us suppose that $\frac{T_{u}}{\Delta(u)} \rightarrow \rho=0$. Clearly, for any $\epsilon>0$,

$$
\pi_{0}(u) \leq \pi_{T_{u}}(u) \leq \pi_{\Delta(u) \epsilon}(u)
$$


Hence, by (31), $\pi_{\Delta(u) \epsilon}(u) \sim \mathcal{H}_{B_{\alpha_{0}}}[0, \epsilon] \mathcal{H}_{B_{\alpha_{0}}} b^{-1 / 2} \sqrt{\pi} \frac{u^{1 / \beta}}{\Delta(u) m(u)} \Psi(m(u))$. Moreover, following [6], we have $\pi_{0}(u) \sim$ $\mathcal{H}_{B_{\alpha_{0}}} b^{-1 / 2} \sqrt{\pi} \frac{u^{1 / \beta}}{\Delta(u) m(u)} \Psi(m(u))$. Thus, using that $\lim _{\epsilon \rightarrow 0} \mathcal{H}_{B_{\alpha_{0}}}[0, \epsilon]=1$, we arrive at

$$
\pi_{T_{u}}(u) \sim \mathcal{H}_{B_{\alpha_{0}}} b^{-1 / 2} \sqrt{\pi} \frac{u^{1 / \beta}}{\Delta(u) m(u)} \Psi(m(u)),
$$

which completes the proof.

5.2. Proof of Theorem 3.2. In view of the proof of Theorem 3.1, using the same notation for $m_{k, l}^{ \pm \epsilon}(u), g_{k, l}(u)$ and $K_{u}$, conditions $\mathbf{P 1}-\mathbf{P} 4$ hold with

$$
\theta_{k, l}\left(u, t, s, t_{1}, s_{1}\right):=\left(\sigma_{1}^{2}\left(\left|s-s_{1}\right|\right)+\sigma_{1}^{2}\left(\left|t-t_{1}\right|\right)\right) \frac{2 \varphi^{2}\left(\tau^{*}\right)^{4 \alpha_{\infty}}}{\left(1+c\left(\tau^{*}\right)^{\beta}\right)^{2}} \frac{\left(m_{k, l}^{-\epsilon}(u)\right)^{2}}{2 \sigma^{2}\left(u^{1 / \beta} \tau^{*}\right)},
$$

where $\sigma_{1}(t)=\frac{1+c\left(\tau^{*}\right)^{\beta}}{\sqrt{2} \varphi\left(\tau^{*}\right)^{2 \alpha_{\infty}}} \sigma(t)$. Thus, following Lemma 5.1,

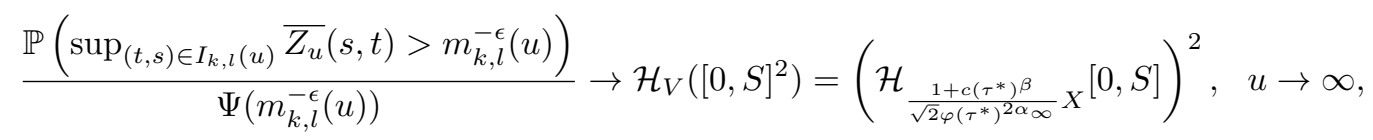

where $V(t, s):=\frac{1+c\left(\tau^{*}\right)^{\beta}}{\sqrt{2} \varphi\left(\tau^{*}\right)^{2 \alpha} \infty}\left(X^{(1)}(t)+X^{(2)}(s)\right)$ with $X^{(1)}, X^{(2)}$ being independent copies of $X$

The rest of the proof goes line-by-line the same as the proof of Theorem 3.1.

5.3. Proof of Theorem 3.3. Similarly to the proof of Theorem 3.1, P1-P4 hold with

$$
\theta_{k, l}\left(u, t, s, t_{1}, s_{1}\right):=\frac{\sigma^{2}\left(\Delta(u)\left|s-s_{1}\right|\right)+\sigma^{2}\left(\Delta(u)\left|t-t_{1}\right|\right)}{\sigma^{2}(\Delta(u))} \frac{\sigma^{2}(\Delta(u))}{2 \sigma^{2}\left(u^{1 / \beta} \tau^{*}\right)}\left(m_{k, l}^{-\epsilon}(u)\right)^{2},
$$

for $(t, s),\left(t_{1}, s_{1}\right) \in[0, S]^{2},(k, l) \in K_{u}$.

In view of Lemma 5.1

$$
\frac{\mathbb{P}\left(\sup _{(t, s) \in I_{k, l}(u) \overline{Z_{u}}}(s, t)>m_{k, l}^{-\epsilon}(u)\right)}{\Psi\left(m_{k, l}^{-\epsilon}(u)\right)} \rightarrow \mathcal{H}_{V}\left([0, S]^{2}\right)=\left(\mathcal{H}_{B_{\alpha_{\infty}}}[0, S]\right)^{2}, \quad u \rightarrow \infty,
$$

where $V(t, s)=B_{\alpha_{\infty}}^{(1)}(t)+B_{\alpha_{\infty}}^{(2)}(s)$ with $B_{\alpha_{\infty}}^{(1)}$ and $B_{\alpha_{\infty}}^{(2)}$ being independent fBms with index $\alpha_{\infty}$. The rest of the proof follows by the same idea as the proof of Theorem 3.1.

5.4. Proof of Theorem 3.4. Similarly to (21), we have

$$
\pi_{T_{u}}^{\inf }(u) \leq \psi_{T_{u}}^{\inf }(u) \leq \pi_{T_{u}}^{\inf }(u)+\mathbb{P}\left(\inf _{t \in\left[0, T_{u} u^{-1 / \beta}\right]} \sup _{s-t \notin E(u)} Z_{u}(s, t)>m(u)\right),
$$

where

$$
\pi_{T_{u}}^{\inf }(u)=\mathbb{P}\left(\inf _{t \in\left[0, T_{u} u^{-1 / \beta}\right]} \sup _{s-t \in E(u)} Z_{u}(s, t)>m(u)\right) .
$$

Due to Lemma 5.6, we get

$$
\begin{aligned}
\mathbb{P}\left(\inf _{t \in\left[0, T_{u} u^{-1 / \beta}\right]} \sup _{s-t \notin E(u)} Z_{u}(s, t)>m(u)\right) & \leq \mathbb{P}\left(\sup _{t \in\left[0, T_{u} u^{-1 / \beta}\right]} \sup _{s-t \notin E(u)} Z_{u}(s, t)>m(u)\right) \\
& =o\left(\frac{u^{1 / \beta}}{\Delta(u) m(u)} \Psi(m(u))\right) .
\end{aligned}
$$


Next we focus on the asymptotics of $\pi_{T_{u}}^{\mathrm{inf}}(u)$.

Case $\varphi=0$ and $\rho \in(0, \infty)$. In order to get the asymptotics of $\pi_{T_{u}}^{\text {inf }}(u)$ we slightly modify arguments used in (29) and (30). Let $D(\rho \pm \epsilon, u)=\left[0,(\rho \pm \epsilon) \frac{\Delta(u)}{u^{1 / \beta}}\right]$ and $F_{l}(u)=\left[\tau_{u}+l \frac{\Delta(u)}{u^{1 / \beta}} S, \tau_{u}+(l+1) \frac{\Delta(u)}{u^{1 / \beta}} S\right]$. Note that functional $\Phi:=\inf$ sup satisfies F1-F2. Using that P1-P4 have been checked in the proof of Theorem 3.1, following Lemma 5.1 , we have

$$
\begin{aligned}
\pi_{T_{u}}^{\inf }(u) & \leq \sum_{l=-N_{S, u}-1}^{N_{S, u}+2} \mathbb{P}\left(\inf _{t \in D(\rho+\epsilon, u)} \sup _{s \in F_{l}(u)} Z_{u}(s, t)>m(u)\right) \\
& \leq \sum_{l=-N_{S, u}-1}^{N_{S, u}+2} \mathbb{P}\left(\inf _{t \in D(\rho+\epsilon, u)} \sup _{s \in F_{l}(u)} \overline{Z_{u}}(s, t)>m_{0, l}^{-\epsilon}(u)\right) \\
& \leq \sum_{l=-N_{S, u}-1}^{N_{S, u}+2} \mathcal{H}_{B_{\alpha_{0}}}^{\inf _{0}}[0, \rho+\epsilon] \mathcal{H}_{B_{\alpha_{0}}}[0, S] \Psi\left(m_{0, l}^{-\epsilon}(u)\right)(1+o(1)) \\
& \leq \mathcal{H}_{B_{\alpha_{0}}}^{\inf }[0, \rho+\epsilon] \mathcal{H}_{B_{\alpha_{0}}}[0, S] \Psi(m(u)) \sum_{l=-N_{S, u}-1}^{N_{S, u}+2} e^{-(b-\epsilon)\left(l m(u) \frac{\Delta(u)}{u^{1 / \beta}} S\right)^{2}}(1+o(1)) \\
& \leq \mathcal{H}_{B_{\alpha_{0}}}^{\inf }[0, \rho+\epsilon] \mathcal{H}_{B_{\alpha_{0}}}(b-\epsilon)^{-1 / 2} \frac{u^{1 / \beta}}{\Delta(u) m(u)} \Psi(m(u)) \int_{-\infty}^{\infty} e^{-x^{2}} d x(1+o(1)) \\
& \sim \mathcal{H}_{B_{\alpha_{0}}}^{\inf }[0, \rho+\epsilon] \mathcal{H}_{B_{\alpha_{0}}}(b-\epsilon)^{-1 / 2} \sqrt{\pi} \frac{u^{1 / \beta}}{\Delta(u) m(u)} \Psi(m(u)),
\end{aligned}
$$

with $N_{S, u}$ and $m_{k, l}^{-\epsilon}(u)$ defined as in the proof of Theorem 3.1. Similarly,

$$
\pi_{T_{u}}^{\mathrm{inf}}(u) \geq \sum_{l=-N_{S, u}}^{N_{S, u}} \mathbb{P}\left(\inf _{t \in D(\rho-\epsilon, u)} \sup _{s \in F_{l}(u)} Z_{u}(s, t)>m(u)\right)-\sum_{i=1}^{2} \Sigma_{i}^{\prime \prime}(u),
$$

with

$$
\Sigma_{i}^{\prime \prime}(u)=\sum_{\left(l, l_{1}\right) \in \Gamma_{i}^{\prime}} \mathbb{P}\left(\inf _{t \in D(\rho-\epsilon, u)} \sup _{s \in F_{l}(u)} Z_{u}(s, t)>m(u) \inf _{t \in D(\rho-\epsilon, u)} \sup _{s \in F_{l_{1}}(u)} Z_{u}\left(s_{1}, t_{1}\right)>m(u)\right), \quad i=1,2,
$$

where $\Gamma_{1}^{\prime}=\left\{\left(l, l_{1}\right),-N_{S, u} \leq l<l_{1}+1 \leq N_{S, u}\right\}$ and $\Gamma_{2}^{\prime}=\left\{\left(l, l_{1}\right),-N_{S, u} \leq l=l_{1}+1 \leq N_{S, u}\right\}$.

Clearly (by the proof of Theorem 3.1)

$$
\begin{aligned}
\sum_{i=1}^{2} \Sigma_{i}^{\prime \prime}(u) & \leq \sum_{i=1}^{2} \sum_{\left(l, l_{1}\right) \in \Gamma_{i}^{\prime}} \mathbb{P}\left(\sup _{t \in D(\rho-\epsilon, u)} \sup _{s \in F_{l}(u)} Z_{u}(s, t)>m(u) \sup _{t \in D(\rho-\epsilon, u)} \sup _{s \in F_{l_{1}}(u)} Z_{u}\left(s_{1}, t_{1}\right)>m(u)\right) \\
& =o\left(\frac{u^{1 / \beta}}{\Delta(u) m(u)} \Psi(m(u))\right)
\end{aligned}
$$

and

$$
\sum_{l=-N_{S, u}}^{N_{S, u}} \mathbb{P}\left(\inf _{t \in D(\rho-\epsilon, u)} \sup _{s \in F_{l}(u)} Z_{u}(s, t)>m(u)\right) \geq \mathcal{H}_{B_{\alpha_{0}}}^{\inf }[0, \rho-\epsilon] \mathcal{H}_{B_{\alpha_{0}}}(b+\epsilon)^{-1 / 2} \sqrt{\pi} \frac{u^{1 / \beta}}{\Delta(u) m(u)} \Psi(m(u)) .
$$

Thus, letting $\epsilon \rightarrow 0$, in view of (33) and (34), we obtain

$$
\pi_{T_{u}}^{\inf }(u) \sim \mathcal{H}_{B_{\alpha_{0}}}^{\inf }[0, \rho] \mathcal{H}_{B_{\alpha_{0}}} b^{-1 / 2} \sqrt{\pi} \frac{u^{1 / \beta}}{\Delta(u) m(u)} \Psi(m(u)) .
$$


Case $\varphi=0$ and $\rho=0$. The idea of proof is based on the observation that

$$
\pi_{\epsilon \Delta(u)}^{\mathrm{inf}}(u) \leq \pi_{T_{u}}^{\mathrm{inf}}(u) \leq \pi_{0}(u)
$$

holds for any $\epsilon>0$ and $u$ sufficiently large. Following $(35), \pi_{\epsilon \Delta(u)}^{\inf }(u)=\mathcal{H}_{B_{\alpha_{0}}}^{\inf }[0, \epsilon] \mathcal{H}_{B_{\alpha_{0}}} b^{-1 / 2} \sqrt{\pi} \frac{u^{1 / \beta}}{\Delta(u) m(u)} \Psi(m(u))(1+$ $o(1))$ as $u \rightarrow \infty$. Using that, due to $[6], \pi_{0}(u)=\mathcal{H}_{B_{\alpha_{0}}} b^{-1 / 2} \sqrt{\pi} \frac{u^{1 / \beta}}{\Delta(u) m(u)} \Psi(m(u))(1+o(1))$ as $u \rightarrow \infty$ and $\lim _{\epsilon \rightarrow 0} \mathcal{H}_{B_{\alpha_{0}}}[0, \epsilon]=1$, the proof is completed.

Case $\varphi \in(0, \infty]$ with $\rho \in[0, \infty)$. The proof of this case can be established in a similar way to that of case $\varphi=0$ and $\rho \in[0, \infty)$.

\section{Appendix}

In the appendix we present the proofs of Lemma 5.1-5.5.

Proof of Lemma 5.1. Since in large part the proof is the same as the proof of Lemma 2 in [6], we present the steps that confirm extension to the class of continuous functionals $\Phi$ that satisfy F1-F2. By the classical transformation, for any $\boldsymbol{k}_{u} \in K_{u}$, we have

$$
\begin{aligned}
& \mathbb{P}\left(\Phi\left(X^{\left(u, \boldsymbol{k}_{u}\right)}\right)>g_{\boldsymbol{k}_{u}}(u)\right) \\
= & \frac{1}{\sqrt{2 \pi} g_{\boldsymbol{k}_{u}}(u)} e^{-\frac{1}{2} g_{\boldsymbol{k}_{u}}^{2}(u)} \int_{\mathbb{R}} e^{w} e^{-\frac{1}{2} \frac{w^{2}}{g_{\boldsymbol{k}_{u}}^{2}(u)}} \mathbb{P}\left(\Phi\left(X^{\left(u, \boldsymbol{k}_{u}\right)}\right)>g_{\boldsymbol{k}_{u}}(u) \mid X_{0}^{\left(u, \boldsymbol{k}_{u}\right)}=g_{\boldsymbol{k}_{u}}(u)-\frac{w}{g_{\boldsymbol{k}_{u}}(u)}\right) d w
\end{aligned}
$$

In light of $\mathbf{F 2}$, we have

$$
\begin{aligned}
& \mathbb{P}\left(\Phi\left(X^{\left(u, \boldsymbol{k}_{u}\right)}\right)>g_{\boldsymbol{k}_{u}}(u) \mid X_{0}^{\left(u, \boldsymbol{k}_{u}\right)}=g_{\boldsymbol{k}_{u}}(u)-\frac{w}{g_{\boldsymbol{k}_{u}}(u)}\right) \\
= & \mathbb{P}\left(\Phi\left(g_{\boldsymbol{k}_{u}}(u)\left(X_{\boldsymbol{t}}^{\left(u, \boldsymbol{k}_{u}\right)}-r_{u, \boldsymbol{k}_{u}}(t) X_{\mathbf{0}}^{\left(u, \boldsymbol{k}_{u}\right)}\right)-g_{\boldsymbol{k}_{u}}^{2}(u)\left(1-r_{u, \boldsymbol{k}_{u}}(\boldsymbol{t})\right)+w\left(1-r_{u, \boldsymbol{k}_{u}}(\boldsymbol{t})\right)\right)>w\right),
\end{aligned}
$$

with $r_{u, \boldsymbol{k}_{u}}(\boldsymbol{t})=\mathbb{E}\left(X_{\boldsymbol{t}}^{\left(u, \boldsymbol{k}_{u}\right)} X_{\mathbf{0}}^{\left(u, \boldsymbol{k}_{u}\right)}\right)$. The reasoning as used in Lemma 2 in [6], in view of (5), F1-F2 and P1-P4, implies that

$$
\Phi\left(g_{\boldsymbol{k}_{u}}(u)\left(X_{\boldsymbol{t}}^{\left(u, \boldsymbol{k}_{u}\right)}-r_{u, \boldsymbol{k}_{u}}(\boldsymbol{t}) X_{\mathbf{0}}^{\left(u, \boldsymbol{k}_{u}\right)}\right)-g_{\boldsymbol{k}_{u}}^{2}(u)\left(1-r_{u, \boldsymbol{k}_{u}}(\boldsymbol{t})\right)+w\left(1-r_{u, \boldsymbol{k}_{u}}(\boldsymbol{t})\right)\right)
$$

weakly converges to $\Phi\left(\sqrt{2} V(\boldsymbol{t})-\sigma_{V}^{2}(\boldsymbol{t})\right)$. Besides, (10) and P3 lead to, for $u$ large enough,

$$
g_{\boldsymbol{k}_{u}}^{2}(u) \mathbb{E}\left(X_{\boldsymbol{t}}^{\left(u, \boldsymbol{k}_{u}\right)}-r_{u, \boldsymbol{k}_{u}}(\boldsymbol{t}) X_{\mathbf{0}}^{\left(u, \boldsymbol{k}_{u}\right)}-X_{\boldsymbol{s}}^{\left(u, \boldsymbol{k}_{u}\right)}+r_{u, \boldsymbol{k}_{u}}(\boldsymbol{s}) X_{\mathbf{0}}^{\left(u, \boldsymbol{k}_{u}\right)}\right)^{2} \leq \mathbb{Q} \sum_{i=1}^{d}\left|s_{i}-t_{i}\right|^{\eta_{i}} .
$$

Thus by $\mathbf{F 1}$ and Fernique inequality, we derive for $u$ large enough and $\delta \in(0,1), w>0$

$$
\begin{aligned}
& \mathbb{P}\left(\Phi\left(g_{\boldsymbol{k}_{u}}(u)\left(X_{\boldsymbol{t}}^{\left(u, \boldsymbol{k}_{u}\right)}-r_{u, \boldsymbol{k}_{u}}(\boldsymbol{t}) X_{\mathbf{0}}^{\left(u, \boldsymbol{k}_{u}\right)}\right)-g_{\boldsymbol{k}_{u}}^{2}(u)\left(1-r_{u, \boldsymbol{k}_{u}}(\boldsymbol{t})\right)+w\left(1-r_{u, \boldsymbol{k}_{u}}(\boldsymbol{t})\right)\right)>w\right) \\
\leq & \mathbb{P}\left(\sup _{\boldsymbol{t} \in M}\left(g_{\boldsymbol{k}_{u}}(u)\left(X_{\boldsymbol{t}}^{\left(u, \boldsymbol{k}_{u}\right)}-r_{u, \boldsymbol{k}_{u}}(\boldsymbol{t}) X_{\mathbf{0}}^{\left(u, \boldsymbol{k}_{u}\right)}\right)-g_{\boldsymbol{k}_{u}}^{2}(u)\left(1-r_{u, \boldsymbol{k}_{u}}(\boldsymbol{t})\right)+w\left(1-r_{u, \boldsymbol{k}_{u}}(\boldsymbol{t})\right)\right)>w\right) \\
\leq & \mathbb{P}\left(\sup _{\boldsymbol{t} \in M} g_{\boldsymbol{k}_{u}}(u)\left(X_{\boldsymbol{t}}^{\left(u, \boldsymbol{k}_{u}\right)}-r_{u, \boldsymbol{k}_{u}}(\boldsymbol{t}) X_{\mathbf{0}}^{\left(u, \boldsymbol{k}_{u}\right)}\right)>(1-\delta) w-a_{1}\right) \\
\leq & a_{2} e^{-a_{3}\left((1-\delta) w-a_{1}\right)^{2}}
\end{aligned}
$$


with $a_{i}, i=1,2,3$ positive constants. The above gives a function that (uniformly) dominates the probability in (36). Then using the dominated convergence theorem, we can get the claim.

Proof of Lemma 5.2. The upper bound follows by the fact that $\sigma^{2}$ is regularly varying at 0 with index $2 \alpha_{0}>0$. Thus we focus on the proof that $\sigma^{2}(t) \geq C_{1} t^{2}$ in a neighbourhood of 0 . For this we use a slight modification of the arguments given in [5].

From AI, there exists $T_{0}>0$ such that for all $T \geq T_{0}$ we have $\sigma(T)>0$ and $\dot{\sigma}^{2}(T)>0$.

Observe that

$$
\sigma\left(T_{0}\right) \sigma(t) \geq \mathbb{E}\left(X\left(T_{0}\right) X(t)\right) \geq 2^{-1}\left(\sigma^{2}\left(T_{0}\right)-\sigma^{2}\left(\left|T_{0}-t\right|\right)\right)
$$

Thus, by Taylor expansion, with $\rho_{t} \in(0, t)$ (and $t>0$ small), we get

$$
\sigma^{2}(T)-\sigma^{2}(T-t)=\dot{\sigma}^{2}\left(T-\rho_{t}\right) t \leq 2 \sigma(T) \sigma(t)
$$

which implies that $\sigma^{2}(t) \geq\left(\frac{\dot{\sigma^{2}\left(T_{0}\right)}}{4 \sigma\left(T_{0}\right)}\right)^{2} t^{2}$ in a neighbourhood of zero.

Proof of Lemma 5.3. Recall that $\sigma_{u}(\tau)=\frac{\sigma\left(u^{1 / \beta} \tau\right)}{\sigma\left(u^{1 / \beta}\right)\left(1+c \tau^{\beta}\right)}$. By UCT (see, e.g., Theorem 1.5.2 in [3]) we have that

$$
\lim _{u \rightarrow \infty} \sigma_{u}(\tau)=\frac{\tau^{\alpha}}{1+c \tau^{\beta}}=g(\tau)
$$

holds uniformly on $(0, S]$ for any $S>0$. Moreover $\tau^{*}=\left(\frac{\alpha_{\infty}}{c\left(\beta-\alpha_{\infty}\right)}\right)^{1 / \beta}$ is the unique maximizer of $g(\tau)$. Further, by Potter's theorem in (see, e.g., [3]), for any $0<\epsilon<\beta-\alpha_{\infty}$ there exists a constant $u_{\epsilon}>0$ such that for all $\tau>1$ and $u>u_{\epsilon}$, we have

$$
\sigma_{u}(\tau) \leq \frac{(1+\epsilon) \tau^{\alpha_{\infty}+\epsilon}}{1+c \tau^{\beta}} \rightarrow 0
$$

as $\tau \rightarrow \infty$. Combing (37) with (38) we conclude that there exist $S_{1}, S_{2}$ such that for sufficiently large $u$ the maximum of $\sigma_{u}(\tau)$ is attained in $\left[S_{1}, S_{2}\right]$ with $0<S_{1}<\tau^{*}<S_{2}<\infty$. Moreover, by AI,

$$
\dot{\sigma_{u}}(\tau) \Rightarrow \dot{g}(\tau), \quad \ddot{\sigma}_{u}(\tau) \Rightarrow \ddot{g}(\tau), \quad \tau \in\left[S_{1}, S_{2}\right]
$$

and $\ddot{g}\left(\tau^{*}\right)<0$.

The above implies that, for each sufficiently large $u$, there exists unique $\tau_{u}$ such that $\tau_{u} \rightarrow \tau^{*}$ as $u \rightarrow \infty, \dot{\sigma_{u}}\left(\tau_{u}\right)=0$ and $\ddot{\sigma}_{u}\left(\tau_{u}\right)<0$. This implies that $\tau_{u}$ is the unique maximizer of $\sigma_{u}(\tau)$, for sufficiently large $u$.

It is straightforward to check that

$$
\frac{g(\tau)}{g\left(\tau^{*}\right)}=1-\frac{B}{2 A}\left(\tau-\tau^{*}\right)^{2}(1+o(1)), \quad \tau \rightarrow \tau^{*},
$$

which combined with (37) and (39) yields (12).

Proof of Lemma 5.4. By direct calculations,

$$
\begin{aligned}
1 & -r_{u}\left(s, t, s_{1}, t_{1}\right)= \\
& =\frac{2 \sigma\left(u^{1 / \beta}(s-t)\right) \sigma\left(u^{1 / \beta}\left(s_{1}-t_{1}\right)\right)+\sigma^{2}\left(u^{1 / \beta}\left|t-t_{1}\right|\right)+\sigma^{2}\left(u^{1 / \beta}\left|s-s_{1}\right|\right)-\sigma^{2}\left(u^{1 / \beta}\left(s-t_{1}\right)\right)-\sigma^{2}\left(u^{1 / \beta}\left(s_{1}-t\right)\right)}{2 \sigma\left(u^{1 / \beta}(s-t)\right) \sigma\left(u^{1 / \beta}\left(s_{1}-t_{1}\right)\right)}
\end{aligned}
$$




$$
=\frac{D_{u}^{(1)}\left(s, t, s_{1}, t_{1}\right)-D_{u}^{(2)}\left(s, t, s_{1}, t_{1}\right)+D_{u}^{(3)}\left(s, t, s_{1}, t_{1}\right)}{2 \sigma\left(u^{1 / \beta}(s-t)\right) \sigma\left(u^{1 / \beta}\left(s_{1}-t_{1}\right)\right)},
$$

where

$$
\begin{aligned}
& D_{u}^{(1)}\left(s, t, s_{1}, t_{1}\right)=\sigma^{2}\left(u^{1 / \beta}\left|t-t_{1}\right|\right)+\sigma^{2}\left(u^{1 / \beta}\left|s-s_{1}\right|\right) \\
& D_{u}^{(2)}\left(s, t, s_{1}, t_{1}\right)=\left(\sigma\left(u^{1 / \beta}(s-t)-\sigma\left(u^{1 / \beta}\left(s_{1}-t_{1}\right)\right)\right)^{2}\right. \\
& D_{u}^{(3)}\left(s, t, s_{1}, t_{1}\right)=\sigma^{2}\left(u^{1 / \beta}(s-t)\right)+\sigma^{2}\left(u^{1 / \beta}\left(s_{1}-t_{1}\right)\right)-\sigma^{2}\left(u^{1 / \beta}\left(s-t_{1}\right)\right)-\sigma^{2}\left(u^{1 / \beta}\left(s_{1}-t\right)\right) .
\end{aligned}
$$

Due to $\mathrm{UCT}$, as $u \rightarrow \infty$,

$$
\frac{\sigma^{2}\left(u^{1 / \beta}\right) t^{2}}{\sigma^{2}\left(u^{1 / \beta} t\right)} \Rightarrow t^{2-2 \alpha_{\infty}}, \quad t \in(0, S], S>0
$$

It follows from mean value theorem and (40) that for $\left|t-t_{1}\right| \leq \delta_{u}, s-t, s_{1}-t_{1} \in E(u)$, with $\theta \in E(u)$,

$$
\begin{aligned}
\frac{D_{u}^{(2)}\left(s, t, s_{1}, t_{1}\right)}{D_{u}^{(1)}\left(s, t, s_{1}, t_{1}\right)} & =\frac{\left(u^{1 / \beta} \dot{\sigma}\left(u^{1 / \beta} \theta\right)\left(s-s_{1}-t+t_{1}\right)\right)^{2}}{\sigma^{2}\left(u^{1 / \beta}\left|t-t_{1}\right|\right)+\sigma^{2}\left(u^{1 / \beta}\left|s-s_{1}\right|\right)} \sim \frac{\alpha_{\infty}^{2} \sigma^{2}\left(u^{1 / \beta} \theta\right)\left(s-s_{1}-t+t_{1}\right)^{2}}{\theta^{2}\left(\sigma^{2}\left(u^{1 / \beta}\left|t-t_{1}\right|\right)+\sigma^{2}\left(u^{1 / \beta}\left|s-s_{1}\right|\right)\right)} \\
& \leq \frac{2 \alpha_{\infty}^{2} \sigma^{2}\left(u^{1 / \beta} \theta\right)\left(\left(s-s_{1}\right)^{2}+\left(t-t_{1}\right)^{2}\right)}{\theta^{2}\left(\sigma^{2}\left(u^{1 / \beta}\left|t-t_{1}\right|\right)+\sigma^{2}\left(u^{1 / \beta}\left|s-s_{1}\right|\right)\right)} \\
& \leq \frac{2 \alpha_{\infty}^{2} \sigma^{2}\left(u^{1 / \beta} \theta\right)\left(s-s_{1}\right)^{2}}{\theta^{2} \sigma^{2}\left(u^{1 / \beta}\left|s-s_{1}\right|\right)}+\frac{2 \alpha_{\infty}^{2} \sigma^{2}\left(u^{1 / \beta} \theta\right)\left(t-t_{1}\right)^{2}}{\theta^{2} \sigma^{2}\left(u^{1 / \beta}\left|t-t_{1}\right|\right)} \rightarrow 0, \quad u \rightarrow \infty .
\end{aligned}
$$

Using Taylor expansion, we have

$$
\begin{aligned}
D_{u}^{(3)}\left(s, t, s_{1}, t_{1}\right)= & u^{1 / \beta} \dot{\sigma^{2}}\left(u^{1 / \beta}\left(s-t_{1}\right)\right)\left(t_{1}-t\right)+\frac{1}{2} u^{2 / \beta} \ddot{\sigma^{2}}\left(u^{1 / \beta} \theta_{1}\right)\left(t-t_{1}\right)^{2} \\
& +u^{1 / \beta} \dot{\sigma^{2}}\left(u^{1 / \beta}\left(s_{1}-t\right)\right)\left(t-t_{1}\right)+\frac{1}{2} u^{2 / \beta} \ddot{\sigma^{2}}\left(u^{1 / \beta} \theta_{2}\right)\left(t-t_{1}\right)^{2} \\
= & \frac{1}{2} u^{2 / \beta} \ddot{\sigma^{2}}\left(u^{1 / \beta} \theta_{1}\right)\left(t-t_{1}\right)^{2}+\frac{1}{2} u^{2 / \beta} \ddot{\sigma^{2}}\left(u^{1 / \beta} \theta_{2}\right)\left(t-t_{1}\right)^{2} \\
& +u^{2 / \beta} \ddot{\sigma^{2}}\left(u^{1 / \beta} \theta_{3}\right)\left(t_{1}-t\right)\left(s-s_{1}+t-t_{1}\right) \\
\leq & u^{2 / \beta}\left(\frac{1}{2} \ddot{\sigma^{2}}\left(u^{1 / \beta} \theta_{1}\right)+\frac{1}{2} \ddot{\sigma}^{2}\left(u^{1 / \beta} \theta_{2}\right)+2 \ddot{\sigma^{2}}\left(u^{1 / \beta} \theta_{3}\right)\right)\left(t-t_{1}\right)^{2} \\
& +2 u^{2 / \beta} \ddot{\sigma^{2}}\left(u^{1 / \beta} \theta_{3}\right)\left(s-s_{1}\right)^{2},
\end{aligned}
$$

where $\theta_{1}, \theta_{2}$ and $\theta_{3}$ are some positive constants satisfying $\frac{\tau^{*}}{2}<\theta_{i}<\frac{3}{2} \tau^{*}, i=1,2,3$, for $u$ sufficiently large. Similarly, in the light of (40), for $\left|t-t_{1}\right| \leq \delta_{u}, s-t, s_{1}-t_{1} \in E(u)$,

$$
\frac{D_{u}^{(3)}\left(s, t, s_{1}, t_{1}\right)}{D_{u}^{(1)}\left(s, t, s_{1}, t_{1}\right)} \rightarrow 0, \quad u \rightarrow \infty .
$$

Hence, the combination of (41) and (42) implies the assertion.

Proof of Lemma 5.5. Substituting $s$ and $s_{1}$ by $t+\tau$ and $t_{1}+\tau_{1}$ respectively yields

$$
\begin{aligned}
& r_{u}\left(t+\tau, t, t_{1}+\tau_{1}, t_{1}\right) \\
& \quad=\frac{\sigma^{2}\left(u^{1 / \beta}\left|t-t_{1}+\tau\right|\right)+\sigma^{2}\left(u^{1 / \beta}\left|t_{1}-t+\tau_{1}\right|\right)-\sigma^{2}\left(u^{1 / \beta}\left|t-t_{1}+\tau-\tau_{1}\right|\right)-\sigma^{2}\left(u^{1 / \beta}\left|t-t_{1}\right|\right)}{2 \sigma\left(u^{1 / \beta} \tau\right) \sigma\left(u^{1 / \beta} \tau_{1}\right)} .
\end{aligned}
$$


Now suppose $t_{1}>t$ and $t_{1}-t>R$ with $R$ a large enough positive constant. Using Taylor expansion at point $t_{1}-t$, we have

$$
\begin{aligned}
& \sigma^{2}\left(u^{1 / \beta}\left(t_{1}-t-\tau\right)\right)+\sigma^{2}\left(u^{1 / \beta}\left(t_{1}-t+\tau_{1}\right)\right)-\sigma^{2}\left(u^{1 / \beta}\left(t_{1}-t+\tau_{1}-\tau\right)\right)-\sigma^{2}\left(u^{1 / \beta}\left(t_{1}-t\right)\right) \\
= & \sigma^{2}\left(u^{1 / \beta}\left(t_{1}-t\right)\right)-\dot{\sigma}^{2}\left(u^{1 / \beta}\left(t_{1}-t\right)\right) u^{1 / \beta} \tau+\frac{1}{2} \ddot{\sigma^{2}}\left(u^{1 / \beta}\left(t_{1}-t+\theta_{1}(u)\right)\right) u^{2 / \beta} \tau^{2} \\
& +\sigma^{2}\left(u^{1 / \beta}\left(t_{1}-t\right)\right)+\dot{\sigma}^{2}\left(u^{1 / \beta}\left(t_{1}-t\right)\right) u^{1 / \beta} \tau_{1}+\frac{1}{2} \ddot{\sigma^{2}}\left(u^{1 / \beta}\left(t_{1}-t+\theta_{2}(u)\right)\right) u^{2 / \beta} \tau_{1}^{2} \\
& -\left(\sigma^{2}\left(u^{1 / \beta}\left(t_{1}-t\right)\right)+\dot{\sigma}^{2}\left(u^{1 / \beta}\left(t_{1}-t\right)\right) u^{1 / \beta}\left(\tau_{1}-\tau\right)+\frac{1}{2} \ddot{\sigma}^{2}\left(u^{1 / \beta}\left(t_{1}-t+\theta_{3}(u)\right)\right) u^{2 / \beta}\left(\tau_{1}-\tau\right)^{2}\right) \\
& -\sigma^{2}\left(u^{1 / \beta}\left(t_{1}-t\right)\right) \\
= & \frac{1}{2} \ddot{\sigma}^{2}\left(u^{1 / \beta}\left(t_{1}-t+\theta_{1}(u)\right)\right) u^{2 / \beta} \tau^{2}+\frac{1}{2} \ddot{\sigma^{2}}\left(u^{1 / \beta}\left(t_{1}-t+\theta_{2}(u)\right)\right) u^{2 / \beta} \tau_{1}^{2} \\
& -\frac{1}{2} \ddot{\sigma}^{2}\left(u^{1 / \beta}\left(t_{1}-t+\theta_{3}(u)\right) u^{2 / \beta}\left(\tau_{1}-\tau\right)^{2},\right.
\end{aligned}
$$

where $\theta_{i}(u), i=1,2,3$ are some constant satisfying $\left|\theta_{i}(u)\right| \leq 2 \tau^{*}, i=1,2,3$ for $u$ large enough. Further, by AI, we have

$$
\begin{aligned}
\left|\frac{\ddot{\sigma}^{2}\left(u^{1 / \beta}\left(t_{1}-t+\theta\right)\right) u^{2 / \beta}}{\sigma\left(u^{1 / \beta} \tau\right) \sigma\left(u^{1 / \beta} \tau_{1}\right)}\right| & =\left|\frac{\ddot{\sigma}^{2}\left(u^{1 / \beta}\left(t_{1}-t+\theta\right)\right) u^{2 / \beta}\left(t_{1}-t+\theta\right)^{2}}{\sigma^{2}\left(u^{1 / \beta}\left(t_{1}-t+\theta\right)\right)} \frac{\sigma^{2}\left(u^{1 / \beta}\left(t_{1}-t+\theta\right)\right)}{\sigma\left(u^{1 / \beta} \tau\right) \sigma\left(u^{1 / \beta} \tau_{1}\right)\left(t_{1}-t+\theta\right)^{2}}\right| \\
& \leq \mathbb{Q} 2 \alpha_{\infty}\left|2 \alpha_{\infty}-1\right| \frac{\left(t_{1}-t+\theta\right)^{2 \alpha_{\infty}+\epsilon}}{\left(\tau^{*}\right)^{2 \alpha_{\infty}+\epsilon}\left(t_{1}-t+\theta\right)^{2}} \\
& \leq \frac{\mathbb{Q}_{1}}{\left(t_{1}-t+\theta\right)^{2-2 \alpha_{\infty}-\epsilon}},
\end{aligned}
$$

where $\mathbb{Q}$ and $\mathbb{Q}_{1}$ are two fixed positive constants, $|\theta| \leq 2 \tau^{*}$ and $0<\epsilon<2-2 \alpha_{\infty}$. Thus we have, as $R \rightarrow \infty$,

$$
\frac{\ddot{\sigma}^{2}\left(u^{1 / \beta}\left(t_{1}-t+\theta\right)\right) u^{2 / \beta}}{\sigma\left(u^{1 / \beta} \tau\right) \sigma\left(u^{1 / \beta} \tau_{1}\right)} \Rightarrow 0,
$$

which implies that for $u$ large enough, $\left|t-t_{1}\right|>R, \tau, \tau_{1} \in E(u)$

$$
r_{u}\left(t+\tau, t, t_{1}+\tau_{1}, t_{1}\right) \Rightarrow 0, \quad R \rightarrow \infty
$$

Next we concentrate on the case of $\left|t-t_{1}\right| \leq R, \tau, \tau_{1} \in E(u)$ with $R$ a positive constant. Applying UCT, we have

$$
\begin{aligned}
r_{u}\left(t+\tau, t, t_{1}+\tau_{1}, t_{1}\right) & \Rightarrow \frac{\left|t-t_{1}+\tau\right|^{2 \alpha_{\infty}}+\left|t_{1}-t+\tau_{1}\right|^{2 \alpha_{\infty}}-\left|t-t_{1}+\tau-\tau_{1}\right|^{2 \alpha_{\infty}}-\left|t-t_{1}\right|^{2 \alpha_{\infty}}}{2 \tau^{\alpha_{\infty}} \tau_{1}^{\alpha_{\infty}}} \\
& \Rightarrow \frac{1}{2}\left(\left|1+\frac{t-t_{1}}{\sqrt{\tau \tau_{1}}}\right|^{2 \alpha_{\infty}}+\left|1+\frac{t_{1}-t}{\sqrt{\tau \tau_{1}}}\right|^{2 \alpha_{\infty}}-2\left|\frac{t-t_{1}}{\sqrt{\tau \tau_{1}}}\right|^{2 \alpha_{\infty}}\right)=f(x),
\end{aligned}
$$

with $\frac{t-t_{1}}{\sqrt{\tau \tau_{1}}}=x$. It is straightforward to check that $\sup _{x \in[\delta, \infty)}|f(x)|<1$ for any $\delta>0$. This completes the proof.

Acknowledgements We thank the referees for their comments and suggestions that highly improved the presentation of the results. We are thankful to Enkelejd Hashorva for proposing the topic of this paper and various related discussions. We also kindly acknowledge partial support from the Swiss National Science Foundation Project 200021-140633/1, and the project RARE -318984 (an FP7 Marie Curie IRSES Fellowship). KD acknowledges partial support by NCN Grant No 2013/09/B/ST1/01778 (2014-2016). 


\section{REFERENCES}

[1] R.J. Adler and J.E. Taylor. Random fields and geometry. Springer Monographs in Mathematics. Springer, New York, 2007.

[2] J. M. P. Albin and G. Samorodnitsky. On overload in a storage model, with a self-similar and infinitely divisible input. Ann. Appl. Probab., 14(2):820-844, 2004.

[3] N. H. Bingham, C. M. Goldie, and J. L. Teugels. Regular variation, volume 27 of Encyclopedia of Mathematics and its Applications. Cambridge University Press, Cambridge, 1989.

[4] K. Dębicki and K. M. Kosiński. On the infimum attained by the reflected fractional Brownian motion. Extremes, 17(3):431-446, 2014.

[5] K. Dębicki. Ruin probability for Gaussian integrated processes. Stochastic Process. Appl., 98(1):151-174, 2002.

[6] A. B. Dieker. Extremes of Gaussian processes over an infinite horizon. Stochastic Process. Appl., 115(2):207-248, 2005.

[7] E. Hashorva, L. Ji, and V.I. Piterbarg. On the supremum of $\gamma$-reflected processes with fractional Brownian motion as input. Stochastic Process. Appl., 123(11):4111-4127, 2013.

[8] J. Hüsler and V.I. Piterbarg. Extremes of a certain class of Gaussian processes. Stochastic Process. Appl., 83(2):257-271, 1999.

[9] J. Hüsler and V.I. Piterbarg. Limit theorem for maximum of the storage process with fractional Brownian motion as input. Stochastic Process. Appl., 114(2):231-250, 2004.

[10] M.R. Leadbetter, G. Lindgren, and H. Rootzén. Extremes and related properties of random sequences and processes, volume 11. Springer Verlag, 1983.

[11] P. Liu, E. Hashorva, and L. Ji. On the $\gamma$-reflected processes with fBm input. Lithuanian Math J., in press, 2015.

[12] I. Norros. A storage model with self-similar input. Queueing Systems Theory Appl., 16(3-4):387-396, 1994.

[13] V.I. Piterbarg. Asymptotic methods in the theory of Gaussian processes and fields, volume 148 of Translations of Mathematical Monographs. American Mathematical Society, Providence, RI, 1996.

[14] V.I. Piterbarg. Large deviations of a storage process with fractional Brownian motion as input. Extremes, 4(2):147-164, 2001.

[15] E. Reich. On the integrodifferential equation of Takács. I. Ann. Math. Statist, 29:563-570, 1958.

Krzysztof Dȩbicki, Mathematical Institute, University of WrocŁaW, Pl. Grunwaldzki 2/4, 50-384 WrocŁaW, Poland

E-mail address: Krzysztof.Debicki@math.uni.wroc.pl

Mathematical Institute, University of WrocŁaw, Pl. Grunwaldzki 2/4, 50-384 WrocŁaw, Poland and Department of Actuarial Science, University of Lausanne, Unil-Dorigny 1015 Lausanne, Switzerland

E-mail address: liupnankaimath@163.com 